\title{
ASTEROID (21) LUTETIA: DISK-RESOLVED PHOTOMETRIC ANALYSIS OF BAETICA REGION
}

\author{
P. H. HASSELMANN ${ }^{1,2}$, M. A. BARUCCI ${ }^{2}$, S. FORNASIER ${ }^{2}$, C. LEYRAT $^{2}$, J. M. CARVANO ${ }^{1}$, \\ D. LAZZARO ${ }^{1}$, AND H. SIERKS ${ }^{3}$
}

\begin{abstract}
${ }^{1}$ Observatį̈œrio Nacional (COAA), Rua General Josï̈œ Cristino 77, Sï œœo Cristï ¿œvï œœo, CEP20921-400, Rio de Janeiro RJ, Brazil.

${ }^{2}$ LESIA, Observatoire de Paris, PSL Research University, CNRS, Univ. Paris Diderot, Sorbonne Paris Citï¡œ, UPMC Univ. Paris 06, Sorbonne Universitï¿œs, 5 Place J. Janssen, 92195 Meudon Principal Cedex, France

${ }^{3}$ Max Planck Institute for Solar System Research, Justus-von-Liebig-Weg 3, 37077, Gï̈œttingen, Germany
\end{abstract}

corresponding author at: hasselmann@on.br

\begin{abstract}
Lutetia has been visited by Rosetta mission on July 2010 and observed with a phase angle ranging from 0.15 to 156.8 degrees. The Baetica region, located at the north pole has been extensively observed by OSIRIS cameras system. Baetica encompass a region called North Pole Crater Cluster (NPCC), shows a cluster of superposed craters which presents signs of variegation at the small phase angle images. For studying the location, we used 187 images distributed throughout 14 filter recorded by the NAC (Narrow Angle Camera) and WAC (Wide Angle Camera) of the OSIRIS system on-board Rosetta taken during the fly-by. Then, we photometrically modeled the region using Minnaert diskfunction and Akimov phase function to obtain a resolved spectral slope map at phase angles of $5^{\circ}$ and $20^{\circ}$. We observed a dicothomy between Gallicum and Danuvius-Sarnus Labes in the NPCC, but no significant phase reddening $\left(-0.04 \pm 0.045 \% \cdot\right.$ microns $\left.^{-1} \mathrm{deg}^{-1}\right)$. In the next step, we applied the Hapke $(2008,2012)$ model for the NAC F82+F22 $(649.2$ $\mathrm{nm})$, WAC F13 (375 nm) and WAC F17 (631.6 nm) and we obtained normal albedo maps and Hapke parameter maps for NAC F82+F22. On Baetica, at $649.2 \mathrm{~nm}$, the geometric albedo is $0.205 \pm 0.005$, the average single-scattering albedo is $0.181 \pm 0.005$, the average asymmetric factor is $-0.342 \pm 0.003$, the average shadow-hiding opposition effect amplitude and width are $0.824 \pm 0.002$ and $0.040 \pm 0.0007$, the average roughness slope is $11.45^{\circ} \pm 3^{\circ}$ and the average porosity is $0.85 \pm 0.002$. We are unable to confirm the presence of coherent-backscattering mechanism. In the NPCC, the normal albedo variegation among the craters walls reach $8 \%$ brighter for Gallicum Labes and $2 \%$ fainter for Danuvius Labes. The Hapke parameter maps also show a dicothomy at the opposition effect coefficients, single-scattering albedo and asymmetric factor, that may be attributed to the maturation degree of the regolith or to compositonal variation. In addition, we compared the Hapke $(2008,2012)$ and Hapke (1993) parameters with laboratory samples and other small-solar system bodies visited by space missions.
\end{abstract}

Asteroid Lutetia, Regoliths, Photometry 


\section{INTRODUCTION}

The asteroid (21) Lutetia was observed during a flyby by Rosetta mission on July 10th, 2010. Rosetta is an ESA cornerstone mission, launched on March 2nd, 2004, composed of two elements: the Orbiter and the lander Philae, with the aim to visit the comet 67P/Churyumov-Gerasimenko. Rosetta spacecraft reached the comet from the heliocentric distance of about $4 \mathrm{AU}$ to start the characterization of the nucleus prior to the delivery of the Philae lander (November 12, 2014) and followed it until its perihelion passage in August 2015 with the end of extended mission on September 2016.

The final choice of the targets (21) Lutetia and (2867) Steins (Barucci et al., 2005) was made only after the launch of the mission and the first orbital correction manï œœuvre. The two asteroids have been selected for their high scientific return and Lutetia in particular because of its large size which was expected to lead to accurate mass and density determinations.

Several instruments were active, resulting into acquiring images and spectrometric observations from the ultraviolet ( $70 \mathrm{~nm}$, by ALICE UV spectrometer) through the visible (by OSIRIS imaging system) and infrared (by the VIRTIS imaging spectrometer) to the millimeter range (0.5-1.3 mm by the MIRO microwave spectrometer), and the radio science investigation (see Barucci et al., in press, for detailed results).

The Rosetta spacecraft flew by (21) Lutetia obtaining resolved images for about 10 hours before the closest approach and revealing an object with a highly complex history. The OSIRIS camera systems (Keller et al. 2007) composed of two cameras (NAC, Narrow Angle Camera and WAC, Wide Angle Camera) observed the asteroid (Sierks et al., 2011) in 21 broad and narrow band filters covering more than $50 \%$ of the surface with spatial scales up to $60 \mathrm{~m} /$ pixel. The rotational period of 8.168 hours and direction of the pole axis were improved (Carry et al. 2010). The global shape with principal axes dimensions of $121 \pm 1 \mathrm{~km} \times 101 \pm 1 \mathrm{~km} \times 75 \pm 13 \mathrm{~km}$ was determined (Sierks et al., 2011), even if a large fraction of the asteroid's southern hemisphere was not visible during the fly-by and consequently the shortest semi-major dimension is not well constrained. From the shape model, an estimation of volume of $(5.0 \pm 0.4) \cdot 10^{5} \mathrm{~km}^{3}$ has been derived and combining it with the mass obtained by the Radio science investigation $1.7 \cdot 10^{18} \mathrm{~kg} \pm 1$ by Pätzold et al. (2011) a bulk density of $3.4 \pm 0.3 \mathrm{~g} \cdot \mathrm{cm}^{-3}$ has been computed.

The north pole is located near a depression that has been produced by multiple impacts called NPCC (North Polar Crater Clusters). The north rotational pole was roughly pointed towards the Sun at the time of the Rosetta encounter and hence high-resolution imaging was restricted by the illumination to one hemisphere. (21) Lutetia shows to have geological complex surface dominate by impact craters, landslides and a diversity set of lineaments (see Thomas et al., 2012, for detailed description). The largest visible depression is the Massilia structure, which is a highly degraded crater-like structure of $57 \mathrm{~km}$ diameter. The surface shows a remarkable structure with boulders and landslides. Its rim appears to have been modified by subsequent impacts. Another dominant feature is the NPCC itself, which forms the most striking structure within the Baetica region. This appears to be one of the youngest surfaces on the object and results of several impacts of varying size, which have overlapped each other.

The relatively low density of smaller impact craters within the NPCC contrasts sharply with the high crater density seen in other regions. Marchi et al. (2012) studied the crater distribution and estimate the age for different regions on Lutetia. Significant amounts of ejecta were also observed on the far side of the impact crater. Detailed results on Lutetia surface characterization are reported and discussed by Barucci et al. (in press). 


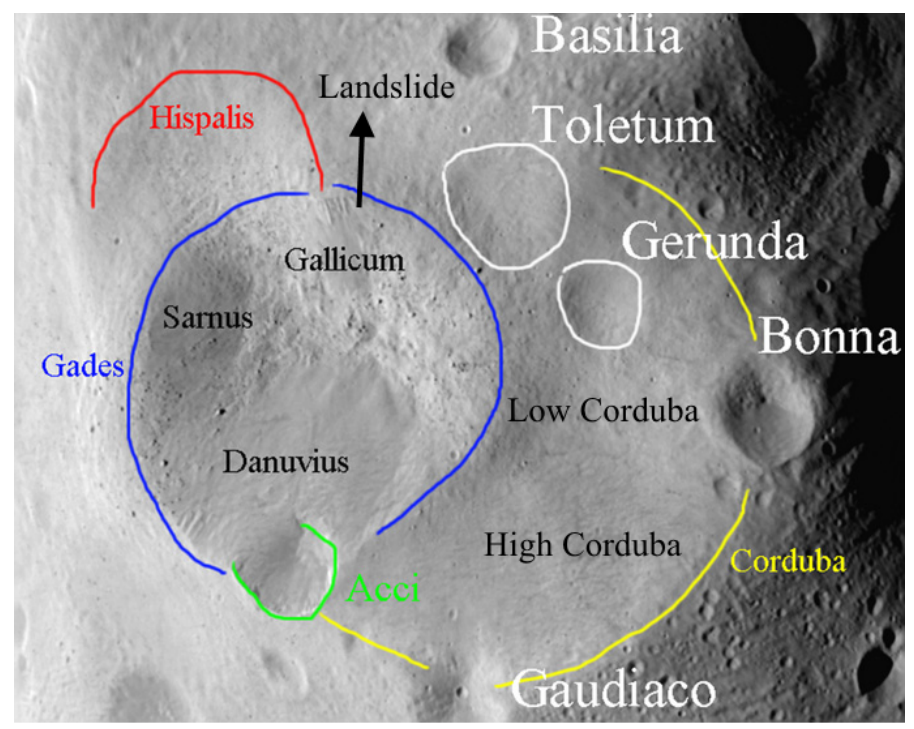

FIGURE 1.1. The geomorphological units of the Baetica Region reproduced from Thomas et al. (2012). Sarnus Labes, Gallicum Labes, Danuvius Labes are the major landslides on NPCC. Gades has diameter $21 \mathrm{~km}$, Corduba - $34 \mathrm{~km}$, Hispalis - $14 \mathrm{~km}$ e Acci - 6 km. On Corduba, we have separated the labelling in the Lowland and Highland according to the topographical slope (we reference Figure 4 in Marchi et al. 2012 for a topographical map).

In this paper we analyze the Baetica region (Figure 1.1) as observed by the OSIRIS camera. We apply empirical and theorical photometric analysis to derive the spectral slope map and semi-physical parameters of the surface. All analysis is done in disk-resolved scale. The region is our main focus of interest due to the identification of some talus of bright material coming down though one of the walls of the NPCC at the small phase angle images (Schulz et al., 2012; Magrin et al., 2012, Thomas et al., 2012), moreover it is the only one to be most extensively covered during all fly-by. Therefore, we are interest to investigate possible space weathering processes (Gaffey, 2010), and for this purpose, we analyze real variegations and parametric variations among Gades, Corduba and surrouding areas.

(21) Lutetia has been observed with a phase angle ranging from 0.15 to 156.85 degrees, a varying resolution of $0.375 \mathrm{~km} /$ pixel to $0.059 \mathrm{~km} /$ pixel and limited incidence angle conditions due the fast fly-by of about $15 \mathrm{~km} / \mathrm{s}$. Only a photometric modeling covering all aspects of the phase curve of an atmosphereless body may provide complete analysis of the (21) Lutetia surface. The main aspect is the opposition effect, a non-linear increase in brightness when the phase angles approaches zero degrees, observed in asteroids for the first time by Gehrels (1956). This has been studied for the connection with two important optical mechanisms of particulate media: The shadow-hiding (e.g., Hapke, 1981, Lumme et al., 1990, Stankevich et al. , 1999) and the coherent-backscattering enhancement (e.g., Muinonen, 1994, Mishchenko et al., 2009). The former is related to the disappearance of mutual shadows among the regolith particles when opposition is near. The latter happens 
when the multiple scattered electromagnetic waves constructively interfere at near zero phase angle. Those mechanisms are intrinsically connected to regolith packing, size distribution, particle shape, inclusions and transparency in a complex relation that it is still subject of on-going research (e.g., Muinonen et al. 2012, Déau et al., 2013). Other aspect is the macroscopic shadowing, a mechanism often significant when a surface is observed at phase angle larger than about 30 degrees and when illumination azimuth is higher. Generally, boulders, micro-craters or micro-irregularities are evoked to explain the hindering of brightness due to the casting of large shadows (Hapke, 1984, Goguen et al. 2010; Shkuratov et al., 2012).

Previous studies on disk-resolved small Solar System bodies have basically relied on a class of models based on Radiative Transfer Equation (RTE) to retrieve semi-physical parameters related to those mechanisms. The Hapke (1981; 1993; 2002, 2008, 2012) models have been the most widespread among the treatment of spacecraft data of atmosphereless bodies of the Solar System. (433) Eros, orbited by NEAR Shoemaker (Clark et al. 2002), Phobos and Deimos, visited by Viking Orbiter (Thomas et al., 1996, Simonelli et al., 1998) and (25143) Itokawa, orbited by Hayabusa (Kitazato et al. 2008) and (4) Vesta, orbited by Dawn (Li et al. 2013b), are examples of small bodies which had their global Hapke parameters obtained. However, only recently, efforts have been taken to derive a spatially resolved Hapke parameters out of disk-resolved data (Spjuth et al., 2012; Sato et al., 2014).

Moving further from the RTE models, there is a class of empirical models that decouples the reflectance dependence of phase angles, the phase function, from the gradient behavior due to local topography, called disk function (e.g., Shkuratov et al.|2011). Generally, those models neglect or roughly describe the multiple-scattering and the macroscopic roughness, making their application restricted to dark smooth surfaces on limited phase angle coverage. A recent application of such models was undertaken by Schröder et al. (2013), where classical light scattering laws as Lambert, Minnaert, Lommel-Seeliger and Akimov were used to photometrically correct images of (4) Vesta, and an empirical phase function was used to fit each single pixel on the illuminated surface.

The OSIRIS camera on-board Rosetta observed (21) Lutetia using 23 filters, however the solar phase angle coverage and number of images is not uniform for all filters. We therefore model the filters following two different approaches. First, for normalizing images of undersampled filters to a standard illumination condition, we tested five scattering laws to find the most suitable one for the photometric correction of topography, in same manner as Schröder et al. (2013). Since the phase dependence can vary throughout (21) Lutetia's surface, the individual fitting of Akimov phase function (Akimov, 1988; Shkuratov et al. 2011) was obtained for each surface element of the shape model, called facet. The spectro-photometry of each facet is derived through the normalization to same a phase angle, allowing a spectral slope map to be obtained. On the second approach, for the filters NAC F82+F22 $(649 \mathrm{~nm})$, WAC F13 $(375 \mathrm{~nm})$ and WAC F17 $(630 \mathrm{~nm})$, where the phase angle coverage was more extensive, we undertake a photometric analysis with Hapke model (Helfenstein and Shepard, 2011; Hapke, 2012) to determine photometric parameters at each facet and thus got their albedo, porosity, macroscopic roughness and opposition surge. Hence, we expect modeling the opposition effect at better precision and verify if the albedo variations at the Baetica regions are connected with different soil properties.

Lastly, on the following sections of this work, we describe the characteristics of data and shape model used on this article (Section 2.1), we specify how the emergence, incidence and azimuth angles were obtained through an image simulator (Section 2.2), we explain the photometric correction method (Section 3.1), we present the color and spectral slope 
maps obtained through the method (Section 3.2) and the locally-resolved Hapke parameters (Section 4). We then conclude and interpret results and their implication on the nature of Baetica albedo variations (Section 5 and 6).

\section{OBSERVATIONS AND METHODS}

2.1. OSIRIS Images and Lutetia shape model. Resolved images of (21) Lutetia started to be taken at 9:30 hours before the closest-approach (CA) at a distance of approximately $500.000 \mathrm{~km}$, reaching some 20 pixels wide. The closest-approach happened at $3168.2 \mathrm{~km}$ with a velocity of $15 \mathrm{~km} / \mathrm{s}$. Several hours later, clear images of Baetica region were taken, at 46 minutes before the CA, on a pixel scale of $0.375 \mathrm{~km} /$ pixel and phase angle of 1.9 degrees. From this point, Baetica is on field-of-view until became a dim limb at 4 minutes after the CA at phase angle of 126 degrees. Table 1 presents the characteristics of the filters and images used in this work.

The OSIRIS obtained 462 images on 27 filter combinations. 234 images were taken with NAC, and 228 with WAC. All images used in this work have quality of level 3 on OSIRIS pipeline calibration, which means they were corrected to dark current, bias, coherent noise, geometry distortion and flat field. The images were calibrated to absolute radiance $\left(W \cdot m^{-2} \cdot \mathrm{nm}^{-1} \cdot s r^{-1}\right)$ and converted to radiance factor (I/F) by dividing them to the incoming solar irradiance given by $F=S_{o} / \pi R^{2}$ at Lutetia heliocentric distance of $R=2.72 \mathrm{UA}$ and solar spectral irradiance $S_{o}\left(\mathrm{~W} \cdot \mathrm{m}^{-2} \cdot \mathrm{nm}^{-1}\right.$, normalized at $\left.1 \mathrm{AU}\right)$. The solar spectral irradiance is set to the central wavelength of each filter combination.

The uncertainties of OSIRIS camera change for each filter, mainly due to uncertainties related to the zero point flux. NAC F16 in the UV have errors about 4-6\%, while NAC and WAC filters in the visible and NIR have just $2 \%$ of uncertainties in respect to the absolute flux. WAC F13, however, have estimated errors of about $8-10 \%$. The internal coherence error for each image, i.e., how much noise is able to mischaracterize the image, are estimated to 1.5-2\% (Magrin et al., 2012; Tubiana et al., 2015).

The shape model for (21) Lutetia was obtained through the stereo-photogrammetric analysis (Jorda et al. 2011, Capanna et al. 2012). The shape model has 3145728 facets and 1579014 vertices, comprising a file of $175 \mathrm{MB}$ (Farnham, 2013). Each facet represent a surface element and has about $0.01 \mathrm{~km}^{2}$. Detailed shape models are essential to undertake a disk-resolved photometric correction.

2.2. Retrieving the Photometric Angles. The photometric angles are obtained through the OASIS simulator (Leyrat et al. 2010 ; Spjuth et al. 2012) developed by L. Jorda of the Laboratoire d'Astrophysique de Marseille. The simulator allows us to recreate absolute flux images of a body on same observational conditions based on a given reflectance model. For irregular shapes, ray-tracing code reconstructs shadows over the surface according to incidence angle. The Figure 2.1 exemplifies the degree of reproduction of OASIS simulator when rendering a image using Lambert law.

The OASIS takes the following information as input:

- Schedule, binning, filter and image size.

- Rotational period and orientation of the rotational axis.

- Target and spacecraft trajectory.

- Spacecraft orientation and instrument placement. 
TABLE 1. Summary of the selected OSIRIS NAC and WAC images acquired during the Lutetia fly-by.

\begin{tabular}{|c|c|c|c|c|c|c|}
\hline Filter & $\begin{array}{c}\text { central } \\
\text { wavelength } \\
(\mathrm{nm})\end{array}$ & $\begin{array}{l}\text { FWHM } \\
(\mathrm{nm})\end{array}$ & $\begin{array}{c}\text { Image range } \\
\text { (2010-07-10 UT) }\end{array}$ & $\begin{array}{c}\text { Phase } \\
\text { range }\left({ }^{\circ}\right)\end{array}$ & Number & $\begin{array}{c}\text { Solar } \\
\text { spectral } \\
\text { irradiance } \\
S_{o} \\
\end{array}$ \\
\hline & & & 15.26 .05 .175 & 0.15 & & \\
\hline \multirow[t]{3}{*}{ NAC F82+F22 } & 649.2 & 84.5 & - & - & 47 & 1.5650 \\
\hline & & & 15.50 .39 .219 & 144.15 & & \\
\hline & & & 14.45 .05 .650 & 3.08 & & \\
\hline \multirow[t]{3}{*}{ NAC F83+F23 } & 535.7 & 62.4 & - & - & 5 & 1.9950 \\
\hline & & & 15.43.27.562 & 61.0 & & \\
\hline & & & 14.44 .58 .330 & 2.95 & & \\
\hline \multirow[t]{3}{*}{ NAC F84+F24 } & 480.7 & 74.9 & - & - & 11 & 2.0600 \\
\hline & & & 15.43.19.535 & 59.03 & & \\
\hline & & & 15.15 .28 .228 & 3.51 & & \\
\hline \multirow[t]{3}{*}{ NAC F88+F28 } & 743.7 & 64.1 & - & - & 7 & 1.2890 \\
\hline & & & 15.43 .43 .881 & 65.1 & & \\
\hline & & & 14.44 .51 .065 & 2.82 & & \\
\hline \multirow[t]{3}{*}{ NAC F16 } & 360.0 & 51.1 & - & - & 7 & 1.0305 \\
\hline & & & 15.43.11.261 & 57.07 & & \\
\hline & & & 14.45 .50 .902 & 3.93 & & \\
\hline \multirow[t]{3}{*}{ NAC F41 } & 882.1 & 65.9 & - & - & 7 & 0.9230 \\
\hline & & & 15.43.43.881 & 67.15 & & \\
\hline & & & 14.45 .43 .923 & 4.15 & & \\
\hline \multirow[t]{3}{*}{ NAC F51 } & 805.3 & 40.5 & - & - & 5 & 1.1180 \\
\hline & & & 15.48.45.539 & 128.65 & & \\
\hline & & & 14.45 .58 .036 & 4.01 & & \\
\hline \multirow[t]{3}{*}{ NAC F61 } & 931.9 & 34.9 & - & - & 5 & 0.8480 \\
\hline & & & 15.49 .01 .668 & 130.41 & & \\
\hline & & & 14.46 .05 .075 & 4.22 & & \\
\hline \multirow[t]{3}{*}{ NAC F71 } & 989.3 & 38.2 & - & - & 9 & 0.7363 \\
\hline & & & 15.44.00.348 & 69.36 & & \\
\hline & & & 15.15 .20 .596 & 0.15 & & \\
\hline \multirow[t]{3}{*}{ WAC F13 } & 375.6 & 9.8 & - & - & 30 & 1.1030 \\
\hline & & & 15.47 .49 .781 & 121.44 & & \\
\hline & & & 15.15.23.117 & 3.18 & & \\
\hline \multirow[t]{3}{*}{ WAC F15 } & 572.1 & 11.5 & - & - & 5 & 1.8280 \\
\hline & & & 15.43 .39 .468 & 63.95 & & \\
\hline & & & 15.15 .25 .358 & 3.22 & & \\
\hline \multirow[t]{3}{*}{ WAC F16 } & 590.7 & 4.7 & - & - & 5 & 1.8180 \\
\hline & & & 15.43.47.143 & 65.91 & & \\
\hline & & & 15.14 .41 .474 & 0.33 & & \\
\hline \multirow[t]{3}{*}{ WAC F17 } & 630.5 & 4.0 & - & - & 39 & 1.6300 \\
\hline & & & 15.54 .40 .168 & 149.61 & & \\
\hline & & & 15.15 .28 .330 & 3.26 & & \\
\hline \multirow[t]{2}{*}{ WAC F18 } & 612.6 & 9.8 & - & - & 5 & 1.7090 \\
\hline & & & 15.43.55.127 & 68.0 & & \\
\hline
\end{tabular}




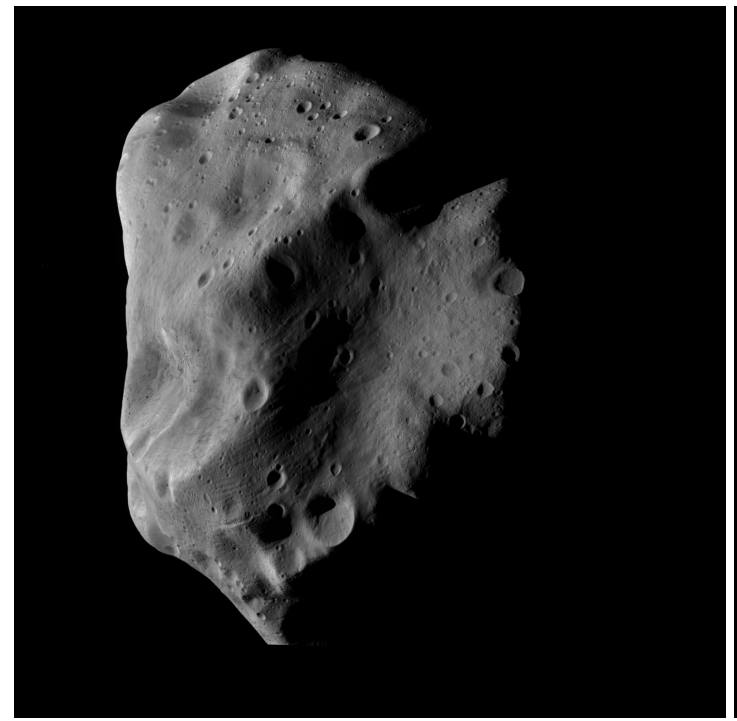

(A)

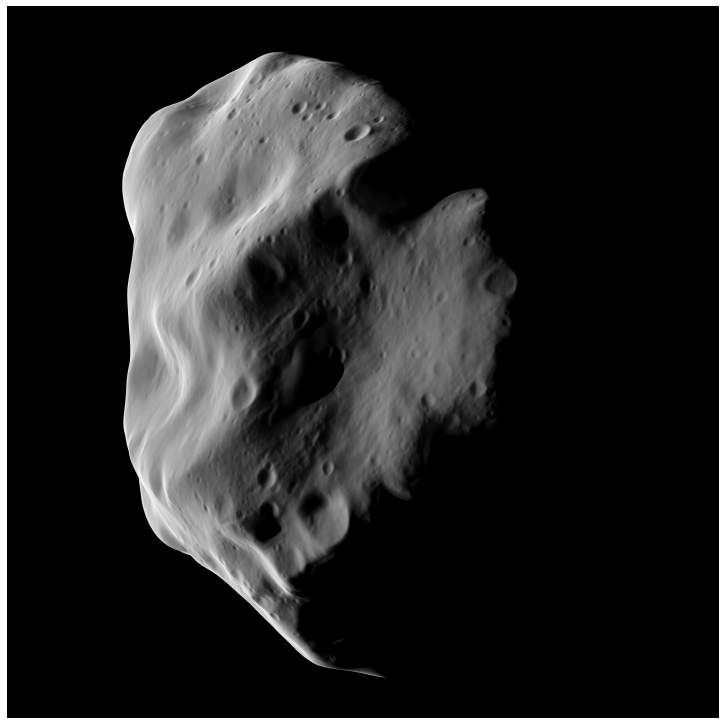

(B)

FIGURE 2.1. (a) Raw image at 15:45:09:210 UT. (b) Rendered image at same time instant by OASIS using Lambert law to recreate the surface reflectance.

- Spacecraft instrument setups as field-of-view, size, gain, coherent noise and pointing.

- Target's shape model.

Then, it provides:

- A rendered image of the object on a specific distance and view;

- a table containing the relation of facets and incidence $(i)$, emergence $(e)$ and azimuth $(\phi)$ angles for the given orbital and rotational configuration;

- a table containing intersection of pixels and facets. The fraction of solid angle $\Omega_{j}$ carried by the facet in one pixel or more is also given.

The trajetory and instrumental information for Rosetta and (21) Lutetia are available in the NAIF SPICE kernels 1 . SPICE kernels are continuously updated on the most accurate position and pointing of the spacecraft as the mission unveils. However, the camera off-set has not been incorporated into instrument pointing, causing shift between real and rendered images, not allowing a perfect link of facets, measured radiance factor and photometric angles. This complication is solved through semi-automatic corregistration, to achieve a sub-pixel overlap in the studied region. An algorithm was written in Python 2.7.5, using the scikit-image package $\left.\right|^{2}$ for such kind of procedure. The user must define at least four control points on exactly same features observed at the real and rendered images, these points are then used to calculate a correspondence matrix used in an affine transformation that shift, rotate and scale the real image to the rendered image. We observe a degraded

\footnotetext{
1 http://www.cosmos.esa.int/web/spice/spice-for-rosetta
}

http://scikit-image.org/ 
matching of the pixels in the border, therefore all facets in the limb $\left(i>80^{\circ}\right.$ and $\left.e>80^{\circ}\right)$ are removed of the analysis.

Thereafter, the OASIS simulator table containing intersection of pixels and facets allows the calculation of radiance factor for each observed facet in the asteroid surface. Those values are then computed through the relation:

$$
(I / F)_{k}=\sum_{j}^{n}(I / F)_{j} \frac{\Omega_{j}}{\Omega_{k}}
$$

where the radiance factor of a given facet $(I / F)_{k}$ is given by the sum of all intersected pixels $j$ weighted by the ratio of the partial solid angle $\Omega_{j}$ over the total solid angle $\Omega_{k}$ of the facet. Thereby, we end with a full catalog of photometric angles and radiance factors for each facet.

The resolved phase curve of Lutetia acquired with NAC F82+F22 is reported in the Figure 2.2 . Most of the data have been acquired at relatively small phase angles, thus we have a good sampling of the opposition surge. If we fit a straight line for the radiance factor of facets observed with phase angle larger than $20^{\circ}$, we measure a increase of the radiance factor of 0.11 due to the non-linear enhancement, which is $\sim 50 \%$ of the albedo at zero phase angle $(0.19 \pm 0.02$, the geometric albedo according to Sierks et al. 2011$)$. The spread of points is due to geometric condition only, the so-called disk profile (Shkuratov et al. 2011), were facets close to limb are obscured in the extreme values of incidence and emergence angles. On Figure $2.2 \mathrm{p}$, we observe a concentration of radiance factor along diagonal that results from the specular behavior of the opposition effect, and outside of it, the diffusive scattering component. Figures 2.2 kd shows that most of Etruria and Baetica areas satisfy the minimum phase angle coverage required to limit the degeneracy among all Hapke parameters $\left(>50^{\circ}\right.$, Fernando et al. $\left.2015 \mathrm{~b}\right)$. Hapke model analysis is reported in the section 4.

\section{SPECTRAL SLOPE}

3.1. Photometric Correction. For the photometric correction, we relied on models where the explicit dependence of phase angles is decoupled from topographic light scattering behavior. Generally, the reliance of this type of modeling is constrained up to phase angle of $\sim 70^{\circ}$. We are aware that resolution per pixel widely varies across the image, and therefore also varies the quality and the amount of variegation we might detect in respect to phase angle.

Radiance factor or apparent albedo is the bi-directional reflectance $r$ depending at arbitrary illumination conditions:

$$
I / F=A_{p}(i, e, \alpha, \lambda)=\pi \cdot r_{(i, e, \alpha, \lambda)}
$$

Then, radiance factor can be separated in two components, the equigonal albedo and the disk brightness contribution:

$$
I / F=A_{e q}(\alpha, \lambda) \cdot D(i, e, \varphi, \bar{\alpha}, \lambda)
$$

The equigonal albedo $A_{e q}$ describes the solar phase angle dependence only, or so-called phase curve. $D(i, e, \varphi, \alpha, \lambda)$ describes brightness distribution over the disk of the resolved 


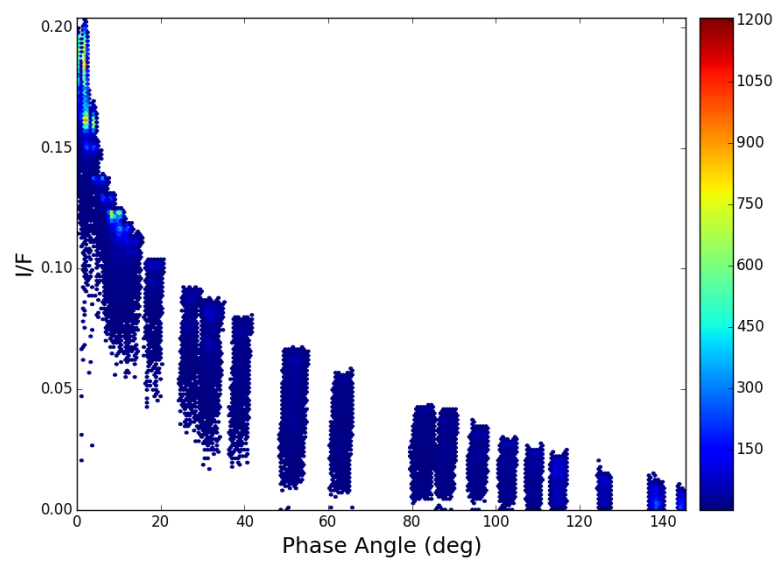

(A)

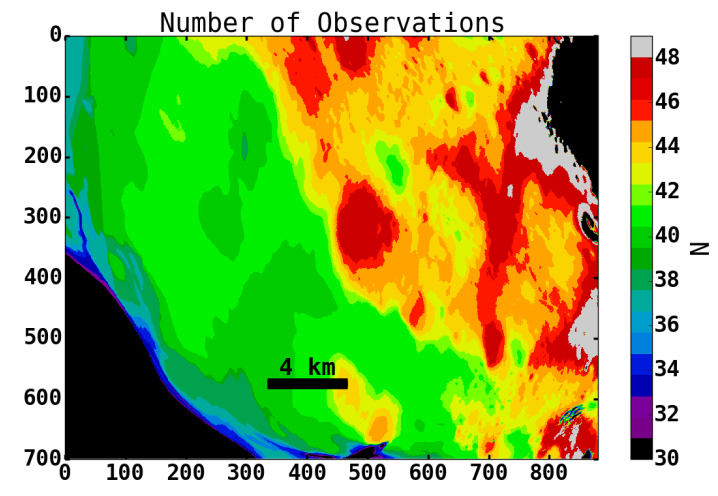

(C)

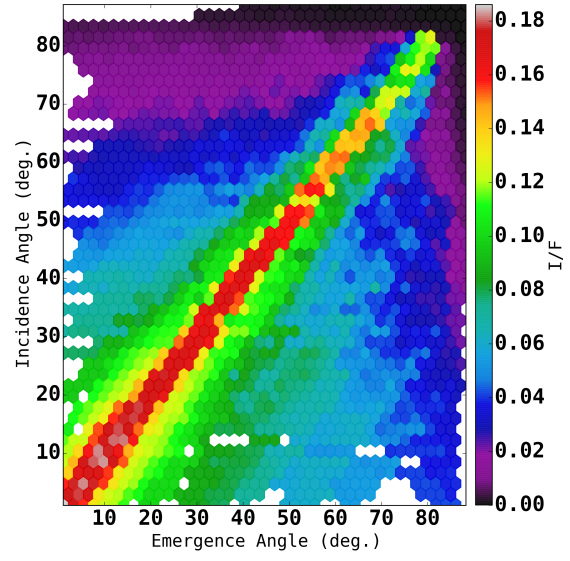

(B)

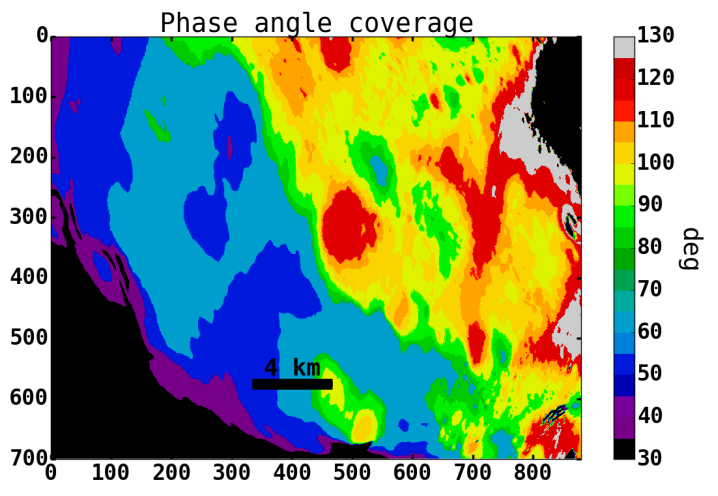

(D)

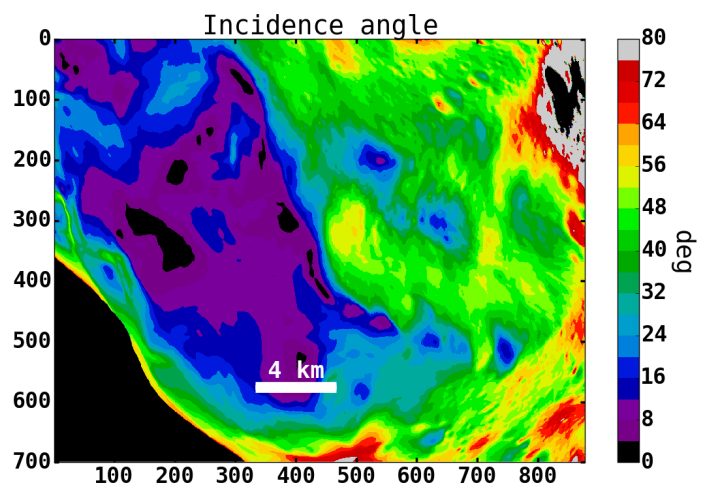

(E)

FIGURE 2.2. Radiance factor of (21) Lutetia in function of the illumination angles. The data was binned on a grid of $40 \times 40 \times 40$ (incidence, emergence, azimuth), with 2 degrees of interval on average. (a) Phase curve of (21) Lutetia in the NAC F82+F22. The color code indicate the number of measurements. (b) Radiance factor as function of $i$ and $e$. Map of (c) the number of observations, (d) the phase angle coverage and (e) incidence angle at Baetica and Etruria regions for $i<80^{\circ}$ and $e<80^{\circ}$ 
TABLE 2. Disk functions

\begin{tabular}{ccc}
\hline Function & Description & Coefficient meaning \\
\hline \hline$D_{L S}=2 \frac{\cos (i)}{\cos (i)+\cos (e)}$ & Lommel-Seeliger law. & - \\
$D_{L}=\cos (i)$ & Lambert law. & - \\
$D_{L L}=2 c \frac{\cos (i)}{\cos (i)+\cos (e)}+(1-c) \cos (i)$ & Lunar-Lambertian (McEwen) law. & L-S to Lambert ratio. \\
$D_{M}=\cos (i)^{k} \cos (e)^{k-1}$ & Minnaert law. & Diversion from lambert law. \\
$D_{O N}^{\dagger}$ & Oren-Nayar model. & Average spike slope. \\
\hline
\end{tabular}

${ }^{\dagger}$ The expression is cumbersome to be written here. We invite readers to check Oren \& Nayar(1993).

object relative to the mirror point fixed at $\alpha$, and it is known as disk function. The disk functions we have implemented are Lambert law, Lommel-Seeliger law (Fairbairn. 2005), Minnaert law (Minnaert, 1941), McEwen or Lunar-Lambertian function (McEwen, 1991) and Oren-Nayar function (Oren \& Nayar 1993) (Table 2). This component is related to limb darkening, topography and also the global surface scattering properties. Further mathematical and qualitative description of photometric definitions was reviewed by Shkuratov et al. (2011). In what follows, the equigonal albedo is also decomposed in two terms:

$$
A_{e q}(\alpha, \lambda)=A_{0}(\lambda) f(\alpha)
$$

where the Normal Albedo $A_{0}$ is the equigonal albedo at $\alpha=0$ and $f(\alpha)$ is the phase function normalized to unity at $\alpha=0$. Retrieving normal albedo is the main goal for characterizing real albedo or color variations over the object landscape. The phase function as proposed by Akimov (Akimov, 1988, Shkuratov et al. 2011) was derived to be applied to a random rough surface area covered by semi-translucent particles:

$$
f(\alpha)=\frac{e^{-\mu_{1} \alpha}+m e^{-\mu_{2} \alpha}}{1+m}
$$

where $m$ is the amplitude of the opposition surge, $\mu_{1}$ and $\mu_{2}$ are related to width of the opposition surge and surface roughness, respectively.

The methodology involved two steps: the disk correction and the facet-by-facet phase curve fitting. First, we took images of phase angle $<90^{\circ}$ and the data was binned into a $40 \times 40 \times 40$ cell grid (about $2^{\circ} \times 2^{\circ} \times\left(0.1^{\circ}-2^{\circ}\right)$ step) of the $i, e$ and $\phi$ angles. The radiance factor was averaged for each cell. This was done to soft the aspect of any albedo variation and bad pixel/facet effect on the disk correction. Then, through the LevenbergMarquardt minimization ${ }^{3}$ (Levenberg, 1944, More, 1978) of the unweighed RMS between measured I/F and equation 3.2, we obtain the $\bar{A}_{e q}(\alpha, \lambda)$ and a disk coefficient for each image. On the next step, we divide the disk function to all measured $\mathrm{I} / \mathrm{F}$ for each image and each facet to obtain $A_{e q}(\alpha, \lambda)$. On the equigonal albedo, we fit each facet using again the Levenberg-Marquardt minimization to obtain the $A_{0}(\lambda)$ and the four parameters of Akimov phase function. We obtain a table contain Normal Albedo and Akimov parameters for each facet and the disk coefficient for each image. The operation is applied for all filters in Table 1. The table of Akimov parameters and Normal Albedos can be replaced in equation 3.3 and 3.4 to reconstruct the Equigonal Albedo for any phase angle.

http://docs.scipy.org/doc/scipy/reference/generated/scipy.optimize.leastsq.html 


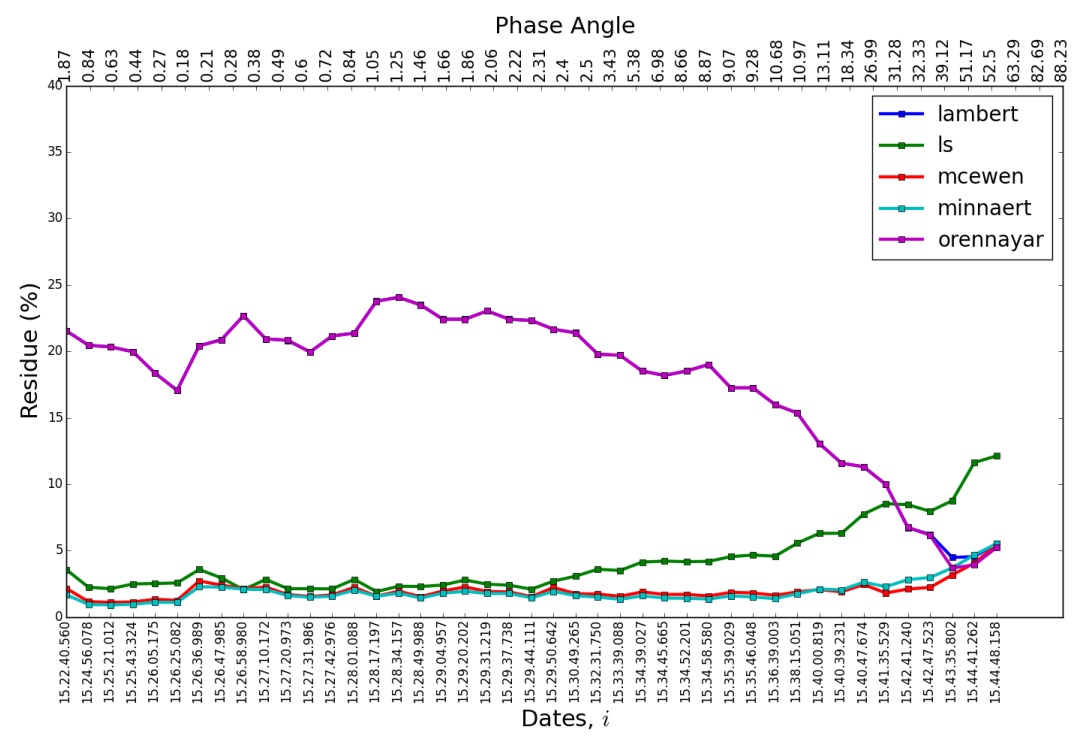

FIGURE 3.1. The normalized RMS (\%) between the modeled and measured radiance factor of each image $i$. The residues of Lunar-Lambertian and Minnaert laws are under $5 \%$.

\subsection{Projected map.}

The disk functions (Table 2) were tested on the NAC F82+F22 images to select the most suitable disk function for describing the photometric profile of Lutetia. As reported in 3.1. the Lunar-Lambertian and Minnaert laws have the smallest residues. Both laws have a hybrid behavior, predicting an uniform disk brightness at zero phase angle and have the Lambert law as limit case $(c=k=1)$. On Figure 3.2 we present the dependence of the disk coefficients of each law as function of phase angle. All coefficients show the same trend: the disk behavior becomes more lambertian as the phase angle increases, as similar in the Moon (McEwen, 1996), Vesta (Schröder et al. 2013) and Tempel 1 (Li et al. 2013a). Lutetia presents a steep Minnaert coefficient slope of $0.005 / \mathrm{deg}$, similar to Vesta, while dark objects like Tempel 1 present a slope of $0.002 /$ deg. Moreover, Lutetia also converges to same Minnaert coefficient as the Moon, Vesta and Temple 1, at $0.5505 \pm$ 0.01 . We find similar behavior for Lunar-Lambertian law. Such behavior is interpreted as weak limb darkening at opposition evolving to a bright limb trend, solely dominated by single scattering. Therefore, we decided to relay on the Minnaert law for the photometric correction of the images of the remaining filters.

The $A_{e q}(\alpha, \lambda)$ for each image and the phase curves for each filter are illustrated at Figure 3.3. The Akimov phase parameters are presented in Table 3 Aside NAC F82+22, WAC F13 and WAC F17, the other filters have a poorer phase angle coverage, and in particular there are no data for phase angle $<3^{\circ}$ and $>60^{\circ}$, and have quite sparse points. Therefore we have fixed the parameter $\mu_{1}$ and hence $A_{0}(\lambda)$ becomes better constrained. All filters have most of their images around phase angles of $5^{\circ}$ and $20^{\circ}$, thus we are able to obtain a better phase curve fitting on these points. We calculated the spectrophotometry 


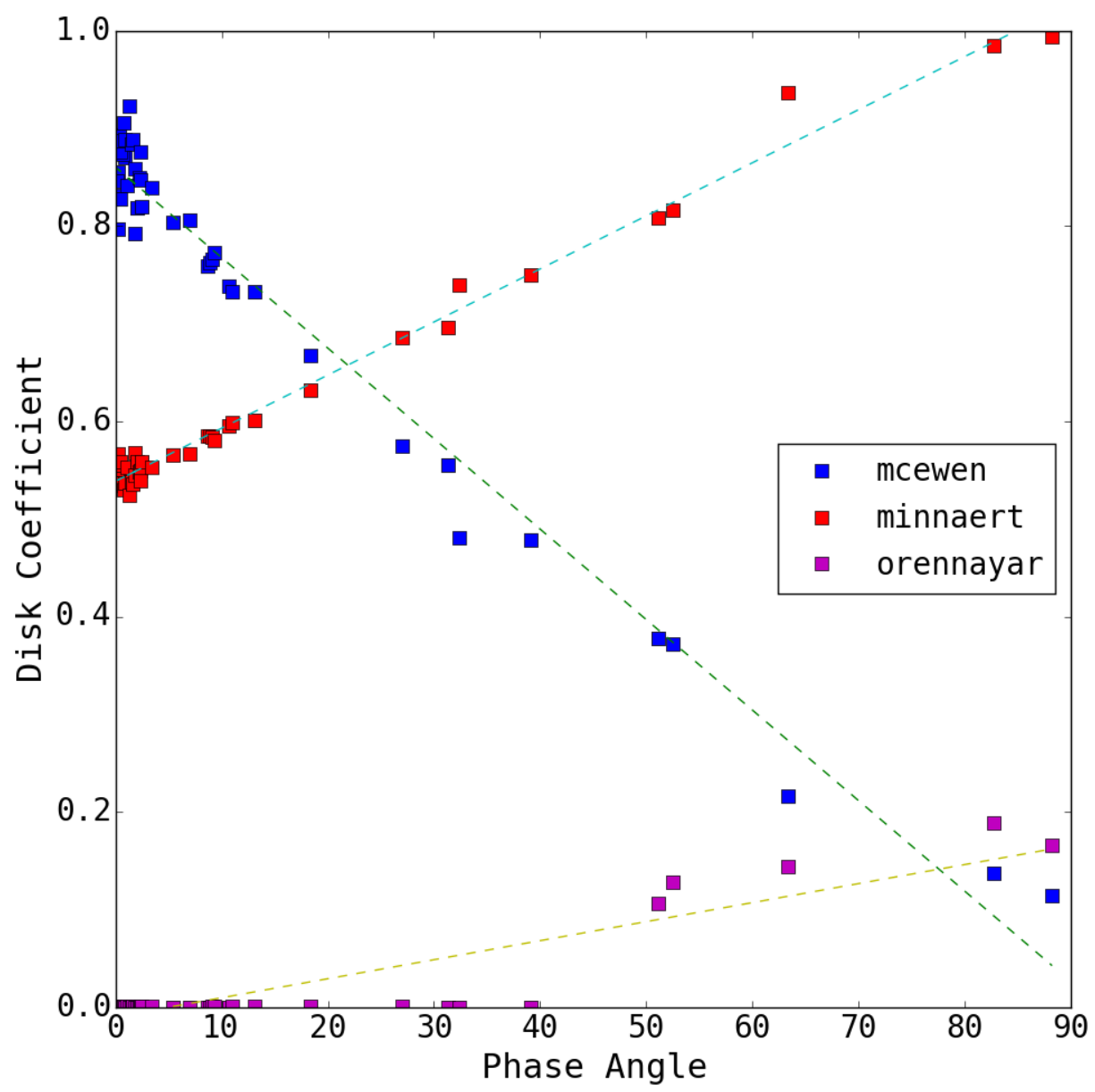

FiguRE 3.2. Disk coefficients of McEwen (Lunar-Lambettian), Minnaert and Oren-Nayar laws as function of phase angles.

$A_{e q}\left(5^{\circ}, \lambda\right)$ and $A_{e q}\left(20^{\circ}, \lambda\right)$ for each filter and a linear equation was fit to each pixel and its spectral slope $\gamma(\alpha)$ computed. We then constructed a disk-resolved projected map of spectral slope $\gamma(\alpha)$ for Baetica region (Figure 3.4). Based on the internal coherence error and an uncertainty on the $i, e$ and $\phi$ angles of 0.5 degrees, we estimate a propagated error to $A_{e q}$ of , at most, $6 \%$. This value is consistent with the variations among neighbouring pixels and previous estimations with OASIS simulator (5\%, Spjuth et al. 2012).

We identify a spectral dichotomy between Gallicum Labes-Low Corduba and Danuvius Labes-Sarnus Labes-High Corbuda. Gallicum and surroundings present a spectral slope of about $18-45 \%\left(4.1-4.8 \% \cdot \mu m^{-1}\right)$ redder than average of $\bar{\gamma}\left(5^{\circ}\right)=3.5 \pm 0.55 \% \cdot \mu m^{-1}$ at phase angle of $5^{\circ}$, while Danuvius Labes present a neutral spectrum of slope of about $15-40 \%\left(2.1-3.0 \% \cdot \mu m^{-1}\right)$ bluer than average. The dichotomy ratio remains similar for 
TABLE 3. Global Akimov phase parameters.

\begin{tabular}{ccccc} 
Filter & $A_{0}$ & $m$ & $\mu_{1}$ & $\mu_{2}$ \\
\hline NAC F82+F22 & 0.195 & 1.32 & 8.55 & 0.678 \\
NAC F83+F23 & 0.179 & 2.02 & - & 0.861 \\
NAC F84+F24 & 0.176 & 2.43 & - & 0.925 \\
NAC F88+F28 & 0.181 & 2.76 & - & 0.857 \\
NAC F16 & 0.165 & 2.28 & - & 0.902 \\
NAC F41 & 0.187 & 2.39 & - & 0.783 \\
NAC F51 & 0.180 & 2.86 & - & 0.849 \\
NAC F61 & 0.200 & 1.39 & - & 0.365 \\
NAC F71 & 0.203 & 2.09 & - & 0.716 \\
WAC F13 & 0.187 & 1.62 & - & 0.791 \\
WAC F15 & 0.175 & 2.70 & - & 0.871 \\
WAC F16 & 0.177 & 2.40 & - & 0.880 \\
WAC F17 & 0.190 & 1.69 & - & 0.768 \\
WAC F18 & 0.174 & 2.66 & - & 0.828
\end{tabular}

the $\gamma\left(20^{\circ}\right)$-map $\left(\bar{\gamma}\left(20^{\circ}\right)=2.9 \pm 0.38 \% \cdot \mu m^{-1}\right)$, but High Corduba becomes bluer by $17-$ $38 \%\left(1.8-2.4 \% \cdot \mu m^{-1}\right)$. Gallicum Labes is covered by a redder and brighter material that extends out of NPCC into the borders of Baetica and Achaia regions. Danuvius Labes, Sarnus Labes and Corduba, on the other hand, are darker and bluer and are confined inside NPCC.

The global phase reddening of spectral slope on Baetica is small and not statistically significant in the considered phase angle range $\left(\frac{\bar{\gamma}\left(20^{\circ}\right)-\bar{\gamma}\left(5^{\circ}\right)}{20^{\circ}-5^{\circ}}=-0.04 \pm 0.045 \% \cdot \mu \mathrm{m}^{-1} \mathrm{deg}^{-1}\right)$. However, specifically on Corduba, we observe a small degree of bluing of -0.053 / $0.066 \pm 0.045 \% \cdot \mu \mathrm{m}^{-1} \mathrm{deg}^{-1}$, not followed by the same behavior on Gallicum, Danuvius or Sarnus. Since High Corbuda and Danuvius Labes are observed at similar incidence and emergence angles, we might rule out any artifact in the photometric correction.

Finally, we clearly see a small bluer spot amid Gallicum Labes connected to the small and smooth landslide we observe in the area (Structure L, details at Thomas et al., 2012). The landslide present the same morphological caracteristics of Danuvius Labes and High Corduba. Gallicum and Danuvius-Sarnus Labes are landslides of very different morphological and photometric characteristics that may have been produced at different temporal events.

\section{HAPKE-PARAMETERS RESOLVED MAP}

4.1. Hapke Model. The Hapke model applied in this work is the latest version by Hapke (2008, 2012). We included some proposed corrections (Helfenstein \& Shepard, 2011) and criticisms (Shkuratov et al. 2012) in respect to the treatement of the CBOE. The model attempts to analytically describe many regimes on the photometric curve of a particulate media based on the most recent theoretical and laboratory results. The model expression for the bi-directional reflectance is: 


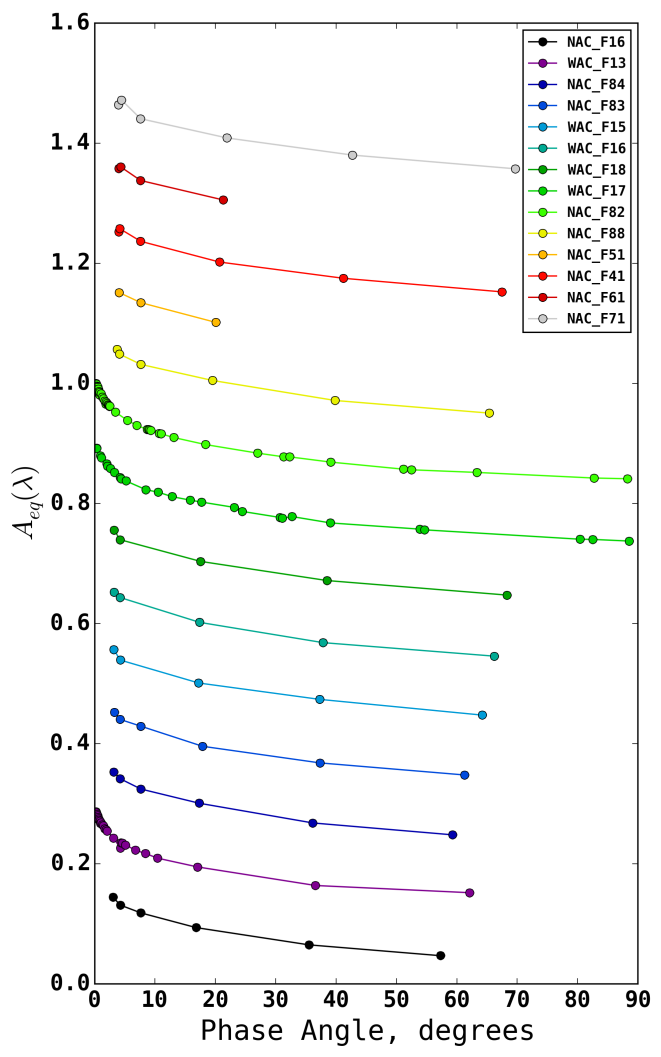

FIGURE 3.3. The $A_{e q}(\alpha, \lambda)$ for each image composing a phase curve for each filter. The curves are shifted by 0.1 unit of radiance factor. Only the images at phase angle under $90^{\circ}$ are used. Over this limit, the photometric correction must strongly relay on a function to correct the macroscopic shadowing.

$$
\begin{gathered}
r_{\left(\mu_{0}, \mu, \alpha\right)}=K \frac{w}{4 \pi}\left(\frac{\mu_{0 e}}{\mu_{e}+\mu_{0 e}}\right)\left[\left(1+B_{S H(\alpha)}\right) P_{(\alpha)}\right. \\
\left.+\left(1+B_{C B\left(\mu_{0}, \mu, \alpha\right)}\right)\left(H_{\left(\mu_{0} / K, w\right)} H_{(\mu / K, w)}-1\right)\right] S_{\left(\mu_{0}, \mu, \alpha\right)}
\end{gathered}
$$

$\mu_{0 e}$ and $\mu_{e}$ are the effectives cosine of incidence and emission angle, involving the topographic correction of the facet. All Hapke explicit parameters are described in the Table 4. As follows, we summarize the mathematical framework for all function in expression 4.1 .

$P_{(\alpha)} \quad$ The single-particle phase function is modeled by the Henyey-Greenstein (HG) functions (Hapke, 2002). The functions are single-term or double-term, depending on the kind of grain is being represented. For double-term function, the asymmetric factor is given as $g_{s c a}=-b \cdot c$. 


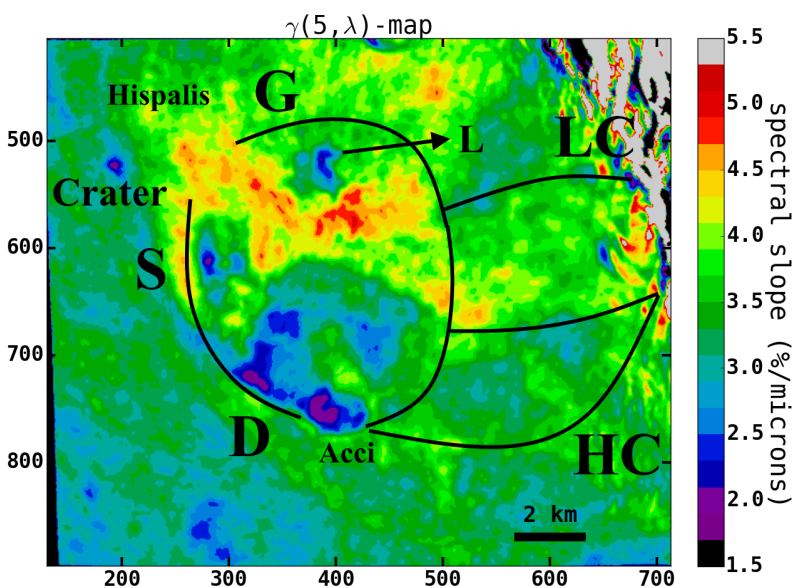

(A)

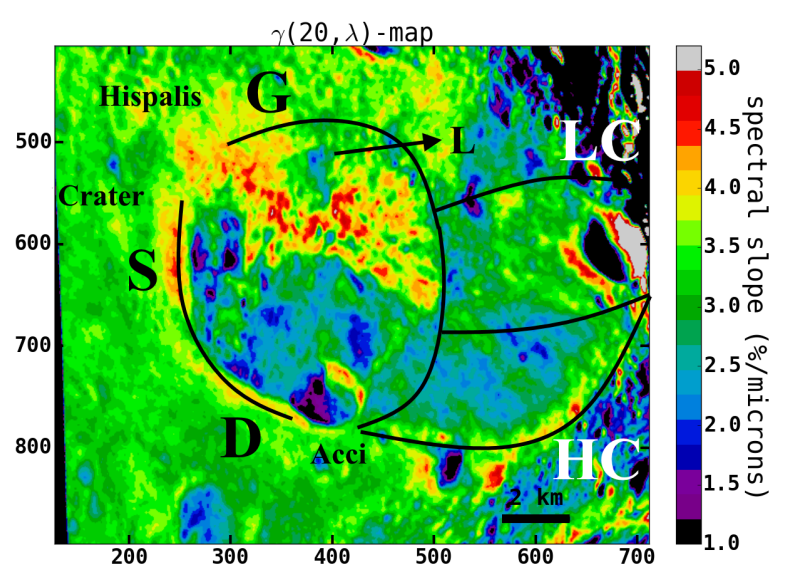

(B)

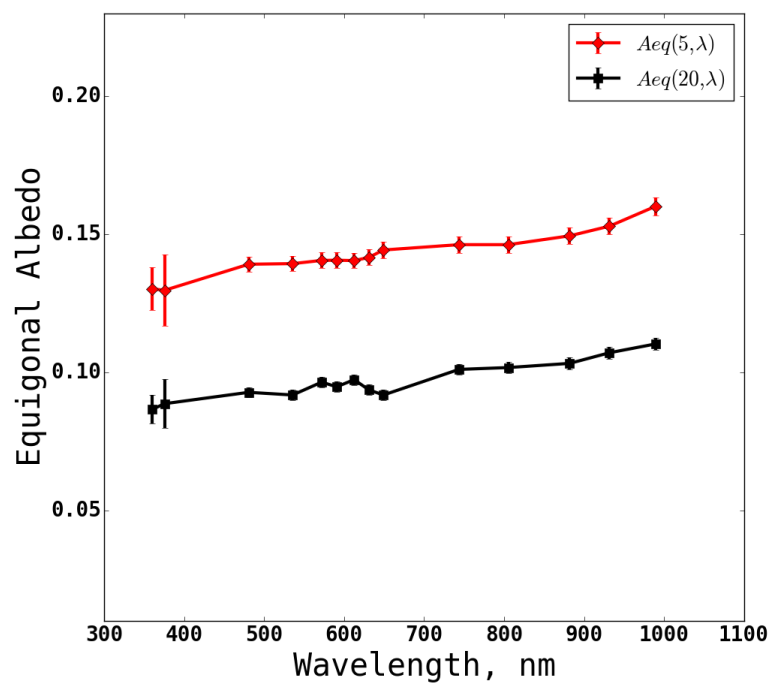

(C)

FIGURE 3.4. Projected spatial map of spectral slope into NAC_201007-10T15.40.39.231Z_ID30_1251276000_F82 image. (a) Spectral slope $\gamma(\alpha)\left(\% \cdot \mu \mathrm{m}^{-1}\right)$ at phase angle of $5^{\circ}$ and (b) of $20^{\circ}$. (c) The global $\bar{A}_{e q}\left(5^{\circ}, \lambda\right)$ and $\bar{A}_{e q}\left(20^{\circ}, \lambda\right)$ spectrophotometry with OSIRIS estimated errors. (G) Gallicum. (D) Danuvius. (S) Sarnus. (L) Landslide. (LC) Low Corduba. (HC) High Corduba.

$B_{S H}\left(\alpha, B s_{0}, h_{s}\right)$ The SHOE function (Section 8, Hapke, 1993). The half-width at halfmaximum (HWHM) of the SHOE is given by $\triangle \alpha_{S H O E}=2 h_{s}$. On our formalism, we explicitly take $B s_{0} \propto \frac{S(0)}{w P(0)}$, as function of the specular component 
TABle 4. Characteristics of the Hapke Parameters.

\begin{tabular}{|c|c|c|}
\hline parameter & Description & Boundaries \\
\hline$w$ & Particle Single-Scattering Albedo (SSA). & $\{0.05,0.5\}$ \\
\hline$b$ & $\begin{array}{l}\text { Coefficients of bi-lobe Heyney-Greenstein function. } b \text { models the } \\
\text { wideness of the back and forward lobes.c represents the partitioned } \\
\text { contribution of each. Both coefficients are correlated by } \\
\qquad c=3.29 \exp \left(-17.4 b^{2}\right)-0.908 \text { (Hapke, 2012). }\end{array}$ & $\{0.1,0.9\}$ \\
\hline$g_{s c a}$ & $\begin{array}{l}\text { Asymmetric factor. Coefficient of the mono-lobe Heyney-Greenstein } \\
\text { function. The average cosine of emergence angle of the particle phase } \\
\text { function. }\end{array}$ & $\{-0.9,0.9\}$ \\
\hline$K$ & $\begin{array}{l}\text { Porosity factor } K=\ln \left(1-1.209 \phi^{2 / 3}\right) / 1.209 \phi^{2 / 3}, \phi \text { is the filling } \\
\text { factor. It is an addition introduced by Hapke (2008) corresponding to } \\
\text { the porosity role in the regolith light scattering. }\end{array}$ & $\{1.0,1.6\}$ \\
\hline$B s_{0}, h_{s}$ & $\begin{array}{l}\text { Amplitude and angular width of the SHOE. } h_{s} \text { relates with } \\
\text { micro-roughness and porosity. } B s_{0} \text { is a function of the specular } \\
\text { component of the particle scattering function. }\end{array}$ & $\begin{array}{l}\{0.0,1.0\} \\
\{0.0,0.52\}\end{array}$ \\
\hline$B c_{0}, h_{c}$ & $\begin{array}{l}\text { Amplitude and angular width of the CBOE. } h_{c}=\frac{\lambda}{2 \pi l} \text {, thus } h_{c} \text { is } \\
\text { inversely proportional to the mean photon path } l . B c_{0} \text { is somehow } \\
\text { connect to the particle scattering matrix. }\end{array}$ & $\begin{array}{l}\{0.0,0.6\} \\
\{0.0,0.2\}\end{array}$ \\
\hline $\bar{\theta}$ & Average macroscopic roughness slope of sub-pixel scale. & $\left\{1^{\circ}, 60^{\circ}\right\}$ \\
\hline
\end{tabular}

$S(0)$ (Hapke, 1993). Therefore $B s_{0}=n S(0)$, where $n$ is a unknown proportional component. We observe that normalizing $B s_{0}$ give more realistic values, i.e., under unity. Finally, the porosity factor $K$ and $h_{s}$ are constrained through the approximative formula $K=1.069+2.109 h_{s}+0.577 h_{s}^{2}-0.062 h_{2}^{2}$ Helfenstein and Shepard, 2011).

$B_{C B}\left(\mu_{0}, \mu, \alpha, B c_{0}, h_{c}\right)$ The original CBOE function by Akkermans et al. (1988) (equation 18, Shkuratov et al. 2012). The HWHM of the CBOE is given by $\Delta \alpha_{C B O E}=$ $0.36 h_{c}$. The CBOE mechanism arises from the multiple-scattering, thus we incorporated the proposition of Helfenstein \& Shepard (2011) to make this function multiplying only the multiple-scattering term in equation 4.1 .

$H(x, w) \quad$ The second-order approximate Ambartsumian-Chandrasekhar function describes the multiple-scattering contribution (equation 13, Hapke. 2002).

$S\left(\mu_{0}, \mu, \alpha\right)$ The shadowing function describes the hindering of brightness due to shadows produced by random irregularities on the medium. The formulation is long, thus we invite the readers to consult it in Hapke (1981; 1993).

The Hapke model is a combination of nonlinear functions with a total of four to eight free parameters where its application depends on the bidirectional reflectance sampling, reflectance precision and illumination coverage of the photometric data. The opposition effect terms $B_{S H}$ and $B_{C B}$ are generally applied only when reflectance is measured for $\alpha \lesssim$ $5^{\circ}$, awhile the shadowing function $S$ is introduced when $i \gtrsim 30^{\circ}$ and $\alpha \gtrsim 60^{\circ}$. However, an outcome of inverse problems of nonlinear models is the lack of unique solutions for a given set of measurements. Usually, some groups of solutions can be discarded based on previous assumptions about the nature of the system. In the case of the Hapke model, the entangling of its parameters, mainly the $w, g_{s c a}$ and $\bar{\theta}$, have been widely criticized for a few 
decades (Helfenstein and Veverka. 1989). Nevertheless Fernando et al. (2013), Fernando et al. (2015a) and Schmidt and Fernando (2015) have recently applied a Bayesian inversion to study the probabilistic density function of each Hapke parameter and their degeneracy. Their study has found that $w, b, c$ and $\bar{\theta}$ follow a unimodal distribution around the solution and the parameters can be described with a mean and standard deviation when uncertainties are lower than about 5\%. Fernando et al. (2015a) state that a phase angles $>90^{\circ}$ and phase angle coverage $>50^{\circ}$ are required to constrain the degeneracy, which is, in fact, satisfied by the OSIRIS data in Baetica region. Since it is not the scope of the work to test once more the validity of the Hapke parameters, we have taken Fernando et al. results into consideration when developing our inversion procedure.

4.2. Inversion procedure. The Hapke modeling involved independently fitting each facet with the L-BFGS-B algorithm (Broyden, 1970; Zhu et al., 1997) available through the Scipy package (Van Der Walt et al. 2011). The L-BFGS-B is a minimization method that allows setting boundaries to the variables and uses up to the second derivative to converge to the closest minima. Table 4 presents the boundaries used for the minimization. First, a global modeling is undertake to the binned data (section 3), as the same fashion applied in Fornasier et al. (2015). The global Hapke parameters is used as initial input for each facet. Then, we fit the facets through computational multiprocessing and we divide them into three groups, for computation speeding up purposes:

- Those observed on the opposition regime (phase angle $<7^{\circ}$ ) and with incidence angles higher enough to cast large shadow at neighboring particles $\left(i>30^{\circ}\right)$. For those, we fit the opposition effect mechanisms $\left(B_{S H}\right.$ and $\left.B_{C B}\right)$ and the shadowing function $S\left(\mu_{0}, \mu, \alpha\right)$. Free parameters are $w, b$ or $g_{s c a}, B_{s 0}, h_{s}, B_{c 0}, h_{c}$ and $\bar{\theta}$.

- Those observed on the opposition regime, but with incidence angles unable to cast large shadows $\left(i<30^{\circ}\right)$. The $S\left(\mu_{0}, \mu, \alpha\right)$ does not diverge from unity in this condition. Then, only the $B_{S H}$ and $B_{C B}$ are fit. Free parameters are $w, b$ or $g_{s c a}$, $B_{s 0}, h_{s}, B_{c 0}$ and $h_{c}$.

- Those not observed at the opposition regime. We fit only $S\left(\mu_{0}, \mu, \alpha\right)$. Free parameters are $w, \bar{\theta}, b$ or $g_{s c a}$.

Due to the coupled nature of the some Hapke parameters, we avoided estimating errors for each parameters separately. When the associated errors to the measurements are small, such as the case for OSIRIS data, the main causes of parametric uncertainties are the undersampling of some regimes in the phase curve and the entangling itself. Therefore, we took the average $\mathrm{I} / \mathrm{F}, i, e$ and $\alpha$ angles for each image to simulate the average conditions of a fitting facet. Then, we monitored the convergence of the error-weighted RMS, first derivative and parameters, using the same initial input. After a specific turning point, where the first derivative becomes the smallest and the minimization procedure scans the minima, we retrieve all the associated Hapke parameters and calculated their standard deviation in respect to the final solution.

In the next step, we performed three independent tests with intent to verify the most suitable particle phase function and the role of $\mathrm{CBOE}$. The tests are:

H1 Hapke model with CBOE and bi-lobe H-G function.

$\mathrm{H} 2 \quad$ Hapke model with $\mathrm{CBOE}$ and single-lobe $\mathrm{H}-\mathrm{G}$ function.

H3 Hapke model without CBOE and with single-lobe $\mathrm{H}-\mathrm{G}$ function.

The CBOE and SHOE functions have similar behavior, both represent a small peak close to small phase angles. Generally, the $\mathrm{CBOE}$ can be solely confirm at the observation 
TABLE 5. Hapke average parameters for three tests on Baetica and Etruria regions at NAC F82+22 (649.5 nm). A global Hapke (1993) test is present for comparison with the values given by the updated model.

\begin{tabular}{ccccc}
\hline & $\mathrm{H} 1$ & $\mathrm{H} 2$ & $\mathrm{H} 3$ & Hapke (1993) \\
\hline \hline CBOE & Yes & Yes & No & No \\
$\mathrm{HG}$ & bi-lobe & single-lobe & single-lobe & single-lobe \\
$A_{0}$ & $0.204 \pm 0.005$ & $0.205 \pm 0.005$ & $0.186 \pm 0.005$ & $0.196 \pm 0.005$ \\
$w$ & $0.241 \pm 0.02$ & $0.181 \pm 0.005$ & $0.182 \pm 0.005$ & $0.238 \pm 0.005$ \\
$g_{s c a}$ & $-0.276 \pm 0.006$ & $-0.342 \pm 0.003$ & $-0.343 \pm 0.006$ & $-0.271 \pm 0.001$ \\
$b, c$ & $-0.191 \pm 0.003,1.45 \pm 0.02$ & - & - & - \\
$B s_{0}$ & $0.872 \pm 0.02$ & $0.824 \pm 0.002$ & $0.829 \pm 0.0025$ & $1.69 \pm 0.006$ \\
$h_{s}$ & $0.086 \pm 0.004$ & $0.040 \pm 0.0007$ & $0.039 \pm 0.00065$ & $0.047 \pm 0.0002$ \\
$B c_{0}$ & $0.396 \pm 0.002$ & $0.072 \pm 0.033$ & - & - \\
$h_{c}$ & $0.038 \pm 0.003$ & $0.060 \pm 0.039$ & - & - \\
$\bar{\theta}$ & $13.5^{\circ} \pm 4.6^{\circ}$ & $11.45^{\circ} \pm 3^{\circ}$ & $11.45^{\circ} \pm 1^{\circ}$ & $29.2 \pm 1^{\circ}$ \\
$1-\phi$ & $0.788 \pm 0.003$ & $0.85 \pm 0.002$ & $0.86 \pm 0.002$ & - \\
\hline$R M S$ & & & & 0.0346 \\
\hline
\end{tabular}

of a sharp negative branch at small phase angles (Shkuratov et al. 2002$)$ in the polarimetric curves or the measurement of dependence of HWHM with wavelength (Akkermans et al. 1988). To test that, a initial input for $B c_{0}$ and $h_{c}$ are set both to $0.05\left(\Delta \alpha_{C B O E}=1^{\circ}\right)$. We expect no large divergence from initial condition unless there is any odd opposition peak not possibly fit solely by the SHOE function. The Table 5 presents the global solutions for each test. At first, we observed how oddly the $B c_{0}$ behaves, reaching almost 0.40 at $\mathrm{H} 1$ and barely diverging at $\mathrm{H} 2 . h_{c}$, otherwise, varies $63 \%$. Since the multiple-scattering term, for $w=0.2$ and $K=1.2$, contributes for just about $15 \%$ in the opposition, the factor of $50 \%$ to which the $B_{c 0}$ modifies the multiple-scattering contribution may not be significant in respect to the other effects, causing the indeterminacy of the parameter.

Although, the addition $B_{C B}\left(\mu_{0}, \mu, \alpha, B c_{0}, h_{c}\right)$ of improves the estimation of the geometric albedo, rising the value to $9 \%$ when comparing $\mathrm{H} 2$ to $\mathrm{H} 3$, placing it closer to the thermal geometric albedo (O'Rourke et al. 2012$)$. Inspecting at the residue across the images at Figure 4.1, however, we observe no large variations at smaller phase angles. H2 and $\mathrm{H} 3$ delivers almost the same percent of residue across the images, and it is overall better than $\mathrm{H} 1$. Therefore, the $B_{S H}$ function describes well the opposition effect without any necessary addition of another function. Therefore, aside the geometric albedo, without any noticeably improvement in the residue comparing $\mathrm{H} 3$ over $\mathrm{H} 2$, CBOE may still considered ambiguous under Lutetia opposition effect at NAC F82+22. Nonetheless, we still choose $\mathrm{H} 2$ to represent the Hapke parameters maps produced in the following subsection. The CBOE parameters may still help interpreting the regolith properties.

\subsection{Analysis for the NAC F82+22 filter.}

The projected Hapke parameters, normal albedo and normalized RMS into NAC_201007-10T15.40.39.231Z_ID30_1251276000_F82 image are shown in the Figure 4.2. Some limb, border and crater effects should be ignored due to the method failing to describe extreme illumination and multiple-scattering on strong convex topographies. We observe 


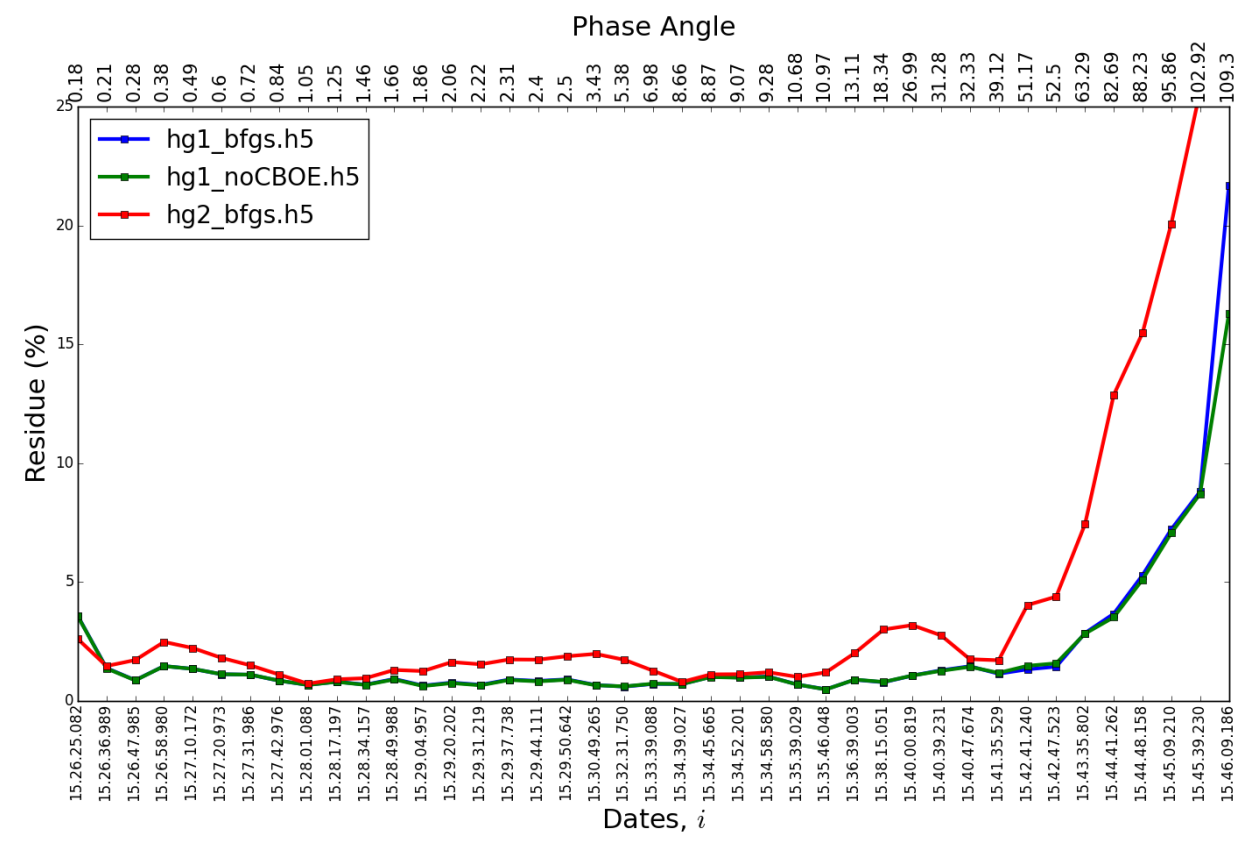

FIGURE 4.1. The normalized RMS between the modeled and measured radiance factor of each image $i$. The residue increases as the surface becomes darker due to the extreme phase angle and as shadows overcome great part of the observed facets.

that Gades and Corduba contains the strongest features in the Baetica region. The average normal albedo of Sarnus Labes, Gallicum Labes are and High Corduba is about $8 \%$ higher than the global average of the region. Danuvius and High Corduba, on the other side, have a normal albedo of about 2-3\% smaller. The differences are also reproduced on the Hapke parameters, where the four regions have their own particular behavior.

Gallicum Labes and Low Corduba, the brighter and rougher NPCC talus on the raw images (Thomas et al. . 2012), are observed on low incidence angle condition, out of the Hapke shadowing function $S$ regime. Its parameters present a broader CBOE and a sharper SHOE, a 33\%-higher SSA and a $B s_{0}$ value closer to average and Danuvius Labes $\left(\bar{B} s_{0}=0.81 \pm 0.03\right)$, which can be interpreted as presence of less opaque, smoother and fine-grained regolith, corresponding to a increase in the internal multiple scattering. This kind of regolith distribution could be related to background soil properties, awhile large settled boulders have insignificant photometric contribution in the scattering. On the spatial distribution of the parameters and on the normal albedo map, we can observe a alignment of bright stripped material coming down from Hispalis to Gallicum and Sarnus, likely related to material once in the bottom of NPCC.

Measured on incidence angles of about $70^{\circ}$, allowing us to estimate $\bar{\theta}$, the Sarnus Labes has also a different behavior from Gallicum and Danuvius. It presents a broad CBOE and SHOE, high $B c_{0}$ and $B s_{0}$ amplitudes, $11 \%$-darker single-scattering albedo and 8\%-higher normal albedo indicates a intermediary regolith where fine-grained particles co-exist with other large opaque particles. Visually, Sarnus is quite similar to Gallicum, which might denote that a broad SHOE could be an effect of the intermediary incidence angle, that turns 

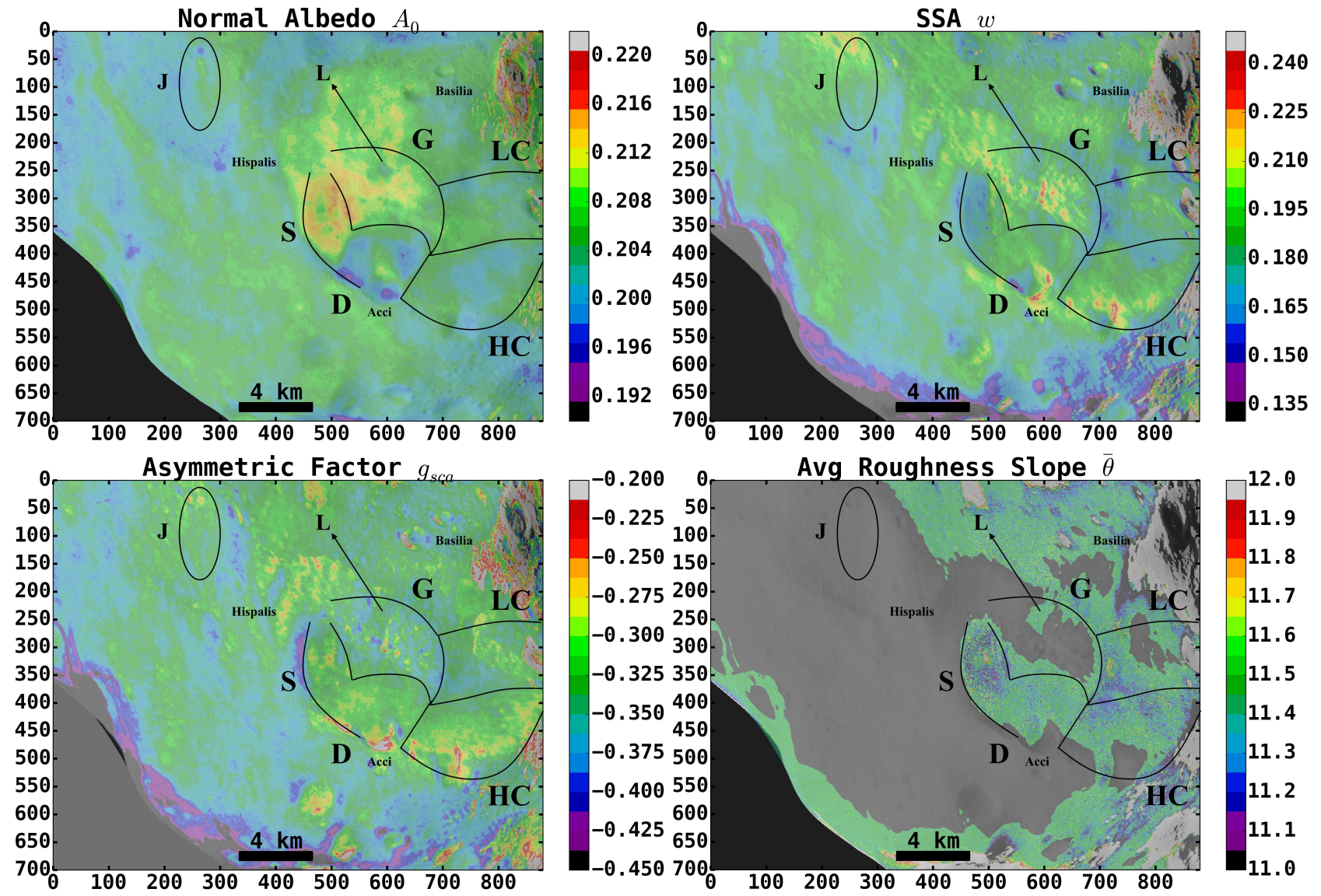

Avg Roughness slope $\bar{\theta}$
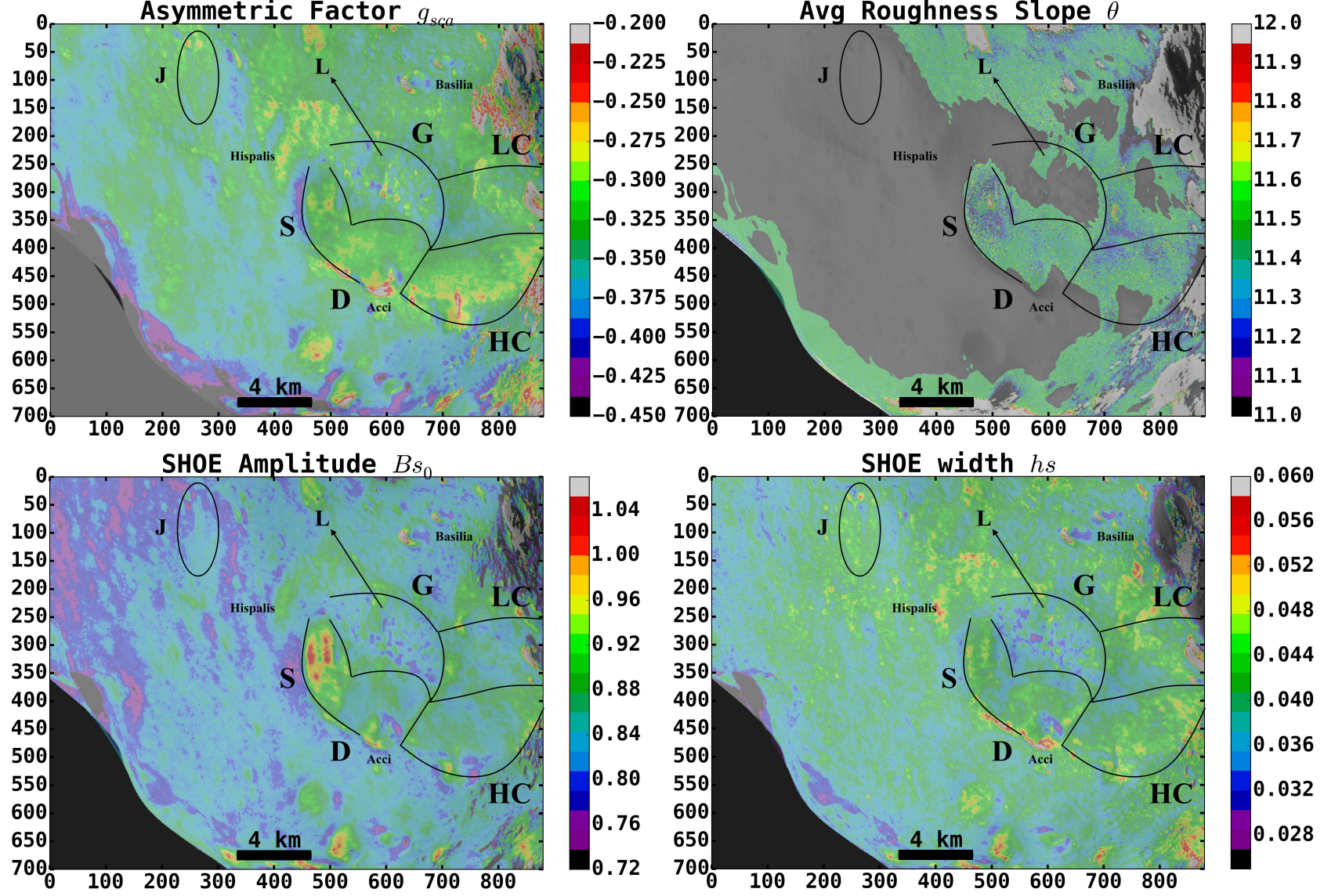

FIGURE 4.2. Resolved Hapke parameters for $650 \mathrm{~nm}$ (NAC F82+F22) projected to NAC_2010-07-10T15.40.39.231Z_ID30_1251276000_F82 image. The projections are labeled according the parameter. Narbonensis region at the right corner returns the most problematic values and highest residue due to the phase angle coverage starting at $34.3^{\circ}$ and the high incidence angles of $80^{\circ}$. (G) Gallicum. (D) Danuvius. (LH) Low Corduba. (HC) High Corduba. (S) Sarnus. (L) Landslide. (J) Ejecta. 
TABLE 6. Hapke parameters of WAC F13 (375.6 nm) and WAC F17 $(630 \mathrm{~nm})$ for Baetica and Etruria region. H2 test applied to both filters.

\begin{tabular}{ccc}
\hline WAC & F13 & F17 \\
\hline \hline CBOE & Yes & Yes \\
HG & single-lobe & single-lobe \\
$A_{0}$ & $0.184 \pm 0.02$ & $0.194 \pm 0.01$ \\
$w$ & $0.165 \pm 0.02$ & $0.179 \pm 0.01$ \\
$g_{s c a}$ & $-0.346 \pm 0.026$ & $-0.349 \pm 0.025$ \\
$b, c$ & - & - \\
$B s_{0}$ & $0.765 \pm 0.015$ & $0.788 \pm 0.013$ \\
$h_{s}$ & $0.034 \pm 0.002$ & $0.035 \pm 0.0013$ \\
$B c_{0}$ & $0.0645 \pm 0.003$ & $0.062 \pm 0.017$ \\
$h_{c}$ & $0.055 \pm 0.014$ & $0.057 \pm 0.07$ \\
$\bar{\theta}$ & $16.9^{\circ} \pm 5^{\circ}$ & $14.04^{\circ} \pm 5^{\circ}$ \\
$1-\phi$ & $0.864 \pm 0.005$ & $0.863 \pm 0.005$ \\
\hline
\end{tabular}

internal shadows more expressive on this lightning condition. The $\bar{\theta} \approx 12^{\circ}$, nonetheless, is close to the average of Baetica region, which does not present any wide variation.

Danuvius Labes and High Corduba are observed at intermediary incidence angles of $45^{\circ}$. Morphologically, Danuvius and High Corduba are smother, absent of larger boulder and presents a wavy-pattern. The wall has a slightly darker normal albedo crossed by a 3\%brighter strip. This strip is probably connected to the Acci crater and it is also reproduced in the SSA and CBOE parameter maps. The most discrepant parameter in the area is the $g_{s c a}$, which is $11 \%$ smaller than average.

Globally for Baetica and part of Etruria, the SHOE mechanism seems completely prevalent on the North pole. The single-scattering albedo is close to the normal albedo, resulting of just about $15 \%$ of contribution by incoherent multiple-scattering in the opposition. The $\bar{\theta}$ is low. The $g_{s c a}$ value represent back-scatter grains, corresponding to rough-surfaced grains (McGuire and Hapke, 1995) or a regolith with significant inter-grain porosity (Shepard and Helfenstein, 2011). Therefore, we can state that Lutetia is largely composed of irregular, opaque and larger-than-wavelength grains under a smooth and porous superficial distribution.

We complement that Etruria region, despite being out of the scope of our work, is partially modeled and presents a interesting bright strip across this region (Structure J). The bright structure starts out of a crater and landslides into a bigger depression. The structure $\mathrm{J}$ is discernible in the $A_{0}, w, B c_{0}, h_{c}$ and porosity maps. It is possibly the ejecta material of a recent crater, which have not yet suffered a spectral attenuation due to space weathering. The material shows similar characteristics to Gallicum Labes and surroundings, but much less brighter.

\subsection{Analysis for the WAC F13 and WAC F17 filters.}

Lutetia was observed with WAC F13 (375 nm) and WAC F17 (630 nm) 30 and 39 times, respectively (Table 1). The images were obtained mainly close to the opposition. The oversampling in this regime allows us a good estimation the Normal Albedo and the opposition 
parameters. Therefore, we applied the same Hapke analysis undertaken to NAC F82+22, using the conditions of test $\mathrm{H} 2$. The Table 6 presents the average Hapke parameters. The Hapke parameter maps are similar to NAC F82+F22. We find a significant spectral variation of the single-scattering albedo and the Normal Albedo (Figure 4.3. We fit a line for each corresponding pixel in the two filters plus the NAC F82+F22, in the same fashion described in section 3.2. The $w$ spectral slope map and the $A_{0}$ spectral slope map (or $\gamma\left(0^{\circ}\right)$ map) presents an average of $\bar{\gamma}_{w}\left(0^{\circ}\right)=6 \pm 1.15 \% \cdot \mu m^{-1}$ and of $\bar{\gamma}\left(0^{\circ}\right)=5.8 \pm 1.35 \% \cdot \mu m^{-1}$, respectively.

The $\gamma\left(0^{\circ}\right)$-map shows the spectral dichotomy of the Gallicum Labes-Low Corduba and Danuvius labes-High Corduba, as previously stated. We measure a phase bluing of $\frac{\bar{\gamma}\left(20^{\circ}\right)-\bar{\gamma}\left(0^{\circ}\right)}{20^{\circ}-0^{\circ}}=-0.145 \pm 0.08 \% \cdot \mu m^{-1} \mathrm{deg}^{-1}$. However, we are dealing with just 3 filters instead of the 14 used in the $\gamma(5, \lambda)$ and $\gamma(20, \lambda)$ maps. If we consider using only the same NAC F82+22, WAC F13 and WAC F17 filters for $\gamma(20, \lambda)$ map, hence the average is then decreased to $\bar{\gamma}\left(20^{\circ}\right)=2.1 \pm 1.92 \% \cdot \mu m^{-1}$. It represents a higher phase bluing $\left(-0.185 \pm 0.13 \% \cdot \mu \mathrm{m}^{-1} \mathrm{deg}^{-1}\right)$, but uncertainties are also increased due to the decrease of filter number, making the phase bluing less statistically significant.

A weak phase bluing, no higher than 3\%, has been reported by Schröder et al. (2014) for some basalt powders between the opposition and mid-phase angles $\left(15^{\circ}-30^{\circ}\right)$. The effect is more evident at $1000 \mathrm{~nm} / 800 \mathrm{~nm}$ ratio for pressed samples and probably connected to the SHOE, since the particles were fully opaque during the experiment. Furthermore, among asteroid surfaces, phase bluing have also been observed for 44 Nysa by Rosenbush et al. (2009) in the U-B and V-R colors. In the U-B, a color opposition effect is observed, indicating a difference in the $\mathrm{OE}$ angular width in respect to the wavelength.

The $w$ spectral slope map shows some distortion due to the border and small craters, but Sarnus Labes bears a redder slope of about 25\% than average. The $w$ and $A_{0}$ spectral slopes seems decoupled, which means that not all spectral wavelength dependence observe in the region is due to single-scattering. Therefore, we expect to observe the dependence of $h_{c}$ with the wavelength, which it is considered an evidence in favor of the presence of CBOE. However, the spectral slope for $h_{c}(\lambda)$ carries a large degree of uncertainty $(16 \pm 13 \%$. $\mu \mathrm{m}^{-1}$ ) and most of the spectro-morphological variations are confined up to $\pm 5.6 \% \cdot \mu \mathrm{m}^{-1}$.

\section{DISCUSSION}

The presence of $C B O E$. Our results are, in respect to the presence of the CBOE, ambiguous. The Hapke CBOE parameters do not improve the fitting and the SHOE only is enough to fit the opposition surge. Although we find an expressive phase bluing in the $A_{0}$ spectral slope map. Belskaya et al. (2010) work on polarimetry of Lutetia was also inconclusive in respect to the existence of coherent-backscattering mechanism. Belskaya et al. showed that Lutetia presents one of broadest negative polarization branch, only (234) Barbara and other four asteroids have wider negative polarization than Lutetia. When comparing Lutetia polarimetric curve with laboratory powder of meteorites, only the carbonaceous chondrites, specially the CV3, show similar minimum of negative branch and inversion angle. Multiple hypothesis have been evoked to explain this phenomena (Shkuratov et al. 1994, for a review), but the shadow-hiding and coherent-backscattering seems to prevail as the main mechanisms (Shkuratov et al. 2002$)$. The connection with such dark meteoritic composition should rule out the contribution of $\mathrm{CBOE}$, since the mechanism is also connect to grain shape and size. Shkuratov et al. (2002) states that scattering of higher orders still 
significantly contribute to negative polarization, even for very dark surfaces. Therefore, the wide negative branch of Lutetia may still be a product of both mechanisms.

A comparison with the Akimov phase parameters and the Hapke parameters. We have calculated the Spearman's rank correlation coefficient among the Akimov phase parameters and Hapke parameters (Figure 5.1). The Spearman's rank measures respectively, from -1 to +1 , the degree of anti-correlation and correlation of two parameters of a sample. As expected, the $A 0_{\text {Akimov }}$ and the $A 0_{\text {Hapke }}$ are strongly correlated. $m_{\text {Akimov }}$ and $w_{1, A k i m o v}$ describe the phase curve non-linear slope, where the first is more related to CBOE term and the latter to SHOE term. We have $w_{2, \text { Akimov }}$ linked to parameters someway related to micro-roughness, mainly $g_{s c a}$ and $h_{s}$. It has been shown that micro-roughness is intrinsically connected to surface porosity (Shepard and Helfenstein, 2007), which is a propriety correlated to $h_{s}$ in Hapke (2008). Thereby the link to porosity $/ h_{s}$ corroborates the interpretation given for $w_{2, \text { Akimov }}$ by Shkuratov et al. (2011).

A comparison with laboratory measurement and Global regolith properties. The precise estimation of Hapke parameters of (21) Lutetia allow us compare it to laboratory samples where their Hapke parameter have been determined. Shepard and Helfenstein (2007) and Shepard and Helfenstein (2011) have tested Hapke model to particulated media of several different characteristics: compression, grain size, roughness, shape and composition. In both paper the authors have accounted for the CBOE, but only in Shepard and Helfenstein (2011) used the same Hapke formalism and accounted for the porosity correction. Beck et al. (2012) have applied the Hapke (1993) model for reflectance phase curve of 8 meteoritic samples. The authors did not include the opposition effect mechanisms, which allows comparisons with just three parameters: $w, g_{s c a}$ and $\bar{\theta}$. While we do not state that any of the samples are, in fact, what Lutetia is composed of, we expect they give us some analog information about the major characteristics of the regolith covering Lutetia in the Baetica region.

None of the laboratory samples of the cited works have matching Hapke parameters we have derived for Baetica. The meteoritic samples were compared to our Hapke (1993) parameters, and likewise, none of them match neither. However, only Cobalt Oxide measured at $700 \mathrm{~nm}(\mathrm{CO} 700)$ and Red Iron Oxide measured at $550 \mathrm{~nm}$ (FE 550) are the particulated media with most similar $w, h_{s}, B s_{0}, \bar{\theta}$ and porosity (their parameters are produced in Table 7).

The CO 700 is described as a dark, flat spectrum and dense powder of $\sim 20 \mu \mathrm{m}$ spheroidal grains. Its SSA, $h_{s}$ and porosity encloses well Lutetia parameters, considering the errors. $\bar{\theta}$ is defined as $16^{\circ}$ in Shepard and Helfenstein (2007) against $0^{\circ}$ in Shepard and Helfenstein (2011). The farther is closer to Lutetia's. On the high-resolution optical images, CO presents some roughness and large grains on its surface, thus $\bar{\theta}=0^{\circ}$ is probably incorrect. On the scanning electron microscope (SEM) images, the grain are indeed more ellipsoidal and of sharp cut shape.

FE 550 is composed of primarily red-spectrum hematite and it is described as composed of shape-complex grains of around 4-10 microns in size. Its SSA, $h_{s}, B s_{0}$ and $b$ are the closest Hapke parameters to Lutetia's. The $\bar{\theta}$ is almost the double, but is encompassed by the average value found on asteroids (Table7). The high-resolution optical images show an very rough surface, populated by large grains and micro shadows. The SEM images present intersected grains of extreme irregular shape. 
However, CO 700 show no similar CBOE parameters. The test presents too high $h_{c}$ of corresponding $\mathrm{HWHM}$ of $27.5^{\circ}$, such large value probably interferes on the estimation of the particle phase function parameters. FE 550, on the other hand, is ambiguous about the CBOE, the RMS is relatively independent of the presence of the mechanism.

A comparison to Masoumzadeh et al. work. Masoumzadeh et al. (2015) has recently undertaken a global photometric analysis of all observed surfaces of (21) Lutetia using Minnaert disk function and Hapke (1993) model. They have analyzed the same OSIRIS NAC F22, NAC F82 and WAC F17 images we used to develop this present work. Their Minnaert analysis has shown an unusual non-linear behavior of the disk function in (21) Lutetia, meaning that Lutetia surface becomes slightly more lambertian at larger phase angles. The $k$ coefficient found in this work for zero phase angle $\left(k_{H 2015}(0)=0.5505 \pm 0.01\right)$ match the one found by them $\left(k_{M 2015}(0)=0.526 \pm 0.002\right)$.

The Hapke (1993) $g_{s c a}, \bar{\theta}$ and $h_{s}$ parameters obtained in this work and Masoumzadeh et al. are in good agreement (Table 7), whilst $w$ and $B s_{0}$ values diverge for just about 6\% and $8 \%$, respectively. These results point to a healthy convergence on both analysis and applied inversion methodology.

When presenting the albedo maps and phase ratios, Masoumzadeh et al. state that no large-scale variegation higher than 7\% aside on NPCC was detected. Our normal albedo and parameter maps support such statements, where large variations on (21) Lutetia are contained in Gades and Corduba craters. However, although no higher than 3\% of the the average normal albedo, we detect some small-scale variations in Etruria as the Structure J.

A comparison with other small solar system bodies. We compiled in the Table7 the global Hapke parameters of Small Solar System Bodies modeled after disk-resolved data. To be able of comparing the global Hapke parameters of Lutetia with other bodies, we also fit the Hapke (1993) model into data (Table 5). Lutetia shares similar $w, g_{s c a}, A_{0}$ and $B s_{0}$ with (243) Ida, a S-type asteroid of $31.4 \mathrm{~km}$ diameter. $\bar{\theta}$ and $A_{0}$ are also close to (941) Gaspra and (433) Eros, both main-belt asteroids of $\mathrm{S}$ group. The resemblance of Lutetia phase curve to S-type asteroids has been previously pointed out by Masoumzadeh et al. (2015). However, we must emphasize we do not state that Lutetia compositionally match to Stype asteroids. The parameters may only indicate that those asteroid share near regolith properties, like grain size distribution, compression and roughness.

Most of the bodies seems to have $B s_{0}>1$, which has been interpreted as necessity to included the CBOE mechanism. None of them present a completely matching opposition effect characteristics $\left(B s_{0}\right.$ and $\left.h_{s}\right)$. The $B s_{0}$ of Lutetia is the second higher after (253) Mathilde among the Main-belt asteroids.

One of few bodies that allows further comparison to our update Hapke parameters is (2867) Steins. (2867) Steins is an E-type, a type of asteroid that generally presents a strong opposition effect (Belskaya et al. 2003). This body was one of the Rosetta asteroid targets and was observed with the OSIRIS camera in similar conditions to (21) Lutetia, having a phase angle that ranged from 0.36 to 141 degrees and varying pixel resolution.Spjuth et al. (2012) used the latest Hapke model to obtain global solution for all shape model facets that were corregistered to rendered images. Then, they fixed all parameters, leaving just one for variation. Hence they obtained residual maps of Hapke parameters for all asteroid observed surface. The CBOE is quite small but it improves the modeling at the opposition regime. Aside the surface porosity value, we find little resemblance of the parameters between both bodies. 


\section{SUMMARY AND CONCLUSION}

We examined images taken in 18 filters by the NAC and WAC cameras of the imaging system OSIRIS on-board Rosetta of the Baetica region and surroundings. The region contains a cluster of superimposed craters called NPCC, where the larger crater walls, called Gallicum Labes, Danuvius Labes, Sarnus Labes, Low Corduba and High Corduba present differences in morphology, texture and boulder distribution (Thomas et al. 2012 ). Aiming to characterize any spectro-photometrical variation among the walls and concerning to investigate the effect of any space weathering process, we undertook a disk-resolved photometric analysis. We applied the latest Hapke model and we were able to draw a spatial distribution map of the parameters. The major results are summarized as:

(1) We observe a spectral dichotomy between Gallicum Labes-Low Corduba and Danuvius Labes-Sarnus Labes-High Corduba. The Gallicum Labes and Low Corduba are around $40 \%$ redder than average spectrum $\left(\bar{\gamma}\left(5^{\circ}\right)=3.5 \pm 0.55 \% \cdot \mu \mathrm{m}^{-1}\right.$, $\bar{\gamma}\left(20^{\circ}\right)=2.9 \pm 0.38 \% \cdot \mu m^{-1}$ ), while Danuvius-Sarnus Labes and High Corduba are $15-40 \%$ bluer. The variegation, otherwise, is no higher than $8 \%$ for GallicumSarnus Labes. Sarnus has a more intriguing behavior, since it shares characteristics of Gallicum and Danuvius Labes, it is spectrally closer to the latter, but it has similar normal albedo to Gallicum Labes. If compared to Psyche Crater in (433) Eros (Clark et al. 2001), the correlation is opposite, the variegation is larger (32-40\%), but spectrally, the dark material at the bottom of the crater and the bright material on the walls, show no more than $4-8 \%$ of difference. Thus, we have confirmed that the variegation and spectral differences between crater walls are significant, as hinted by the small phase angle images of (21) Lutetia. We emphasize that the compositional nature of (21) Lutetia is not accessed by our spectral range or photometric analysis. Lutetia shows a featureless spectra with varying spectral slope. Barucci et al. (2012) have previously concluded that the surface composition is ambiguous, possibly a chondritic mixture of carbonaceous and enstatite.

(2) Gallicum Labes and Low Corduba have the brightest wall $\left(\bar{A}_{0}=0.214 \pm 0.005\right)$ with accentuated asymmetric factor $\left(\bar{g}_{s c a}=-0.35 \pm 0.5\right)$ and with sharper SHOE $\left(\bar{B} s_{0}=0.81 \pm 0.03, h_{s}=0.029 \pm 0.002\right)$ and broader $\operatorname{CBOE}\left(\bar{B} c_{0}=0.10 \pm 0.004\right.$, $\left.h_{c}=0.07 \pm 0.002\right)$. Danuvius Labes and High Corduba, on the other hand, are darker $\left(\bar{A}_{0}=0.199 \pm 0.005\right)$, slightly less back-scatter $\left(\bar{g}_{s c a}=-0.30 \pm 0.1\right)$ with narrower CBOE $\left(\bar{B} c_{0}=0.065 \pm 0.002, h_{c}=0.056 \pm 0.003\right)$ with average SHOE $\left(\bar{B} s_{0}=0.83 \pm 0.02, h_{s}=0.043 \pm 0.001\right)$. The average roughness slope $(\bar{\theta}=11-$ $12^{\circ}$ ) for all the computed areas indicates a smooth terrain.

(3) We have not detected any expressive global phase reddening or bluing on Baetica $\left(-0.04 \pm 0.045 \% \cdot \mu \mathrm{m}^{-1} \mathrm{deg}^{-1}\right)$ between phase angles of $5^{\circ}$ and $20^{\circ}$. The phase angle range between the two sets of images is small to uncover the effect . Locally, we find hint of a slight phase bluing on High Corduba $(-0.053 /-0.066 \pm$ $\left.0.045 \% \cdot \mu m^{-1} \mathrm{deg}^{-1}\right)$. The Highland is connected to a smooth and and darker terrain with different grain distribution than Gallicum Labes.

(4) Measurements with VIRTIS (Coradini et al. 2011) and MIRO (Gulkis et al. 2012) instruments reported very low thermal inertia of $20-30 \mathrm{~J} \cdot \mathrm{m}^{-2} \cdot \mathrm{K}^{-1} \cdot \mathrm{s}^{-\frac{1}{2}}$ or even less. The range is consistent with highly porous upper regolith layer of $1-3 \mathrm{~cm}$ thick. A roughness modeling by Keihm et al. (2012) affirms that a surface composed of $50 \%$ of unresolved craters of $1 \mathrm{~cm}$ or larger are necessary to correct the MIRO-VIRTIS measurement offset. Therefore, the thermal data points to a 
porous regolith layer with an amount of sub-pixel roughness. Our Hapke modeling of $85 \%$ porosity and $11.5^{\circ}$ of average roughness slope, however, is partially consistent with this interpretation. Our $\bar{\theta}$ indicates a smooth surface, but it is not representative of the whole Lutetia surface, since we model only Baetica and part of the Etruria region. However, our global Hapke (1993) modeling returns $\bar{\theta}=29^{\circ}$, a much rougher and compatible surface. This parameter is influenced by the inclusion of the rougher Narbonensis region, which a preliminary modeling indicated $\bar{\theta}$ ranging from $20^{\circ}$ to $30^{\circ}$.

(5) We compared our Hapke parameters with laboratory samples and other disk-resolved small bodies of the Solar System. None of the bodies completely match Baetica, although the global Hapke (1993) parameters of Lutetia match some S-type asteroids. However, as Lutetia spectrum diverges from this taxonomic type, the similarities may only be related to the state of the regolith. Comparing with laboratory, we conclude that Cobalt Oxide $(700 \mathrm{~nm})$ and Red Iron Oxide $(550 \mathrm{~nm})$ are the best analogs for the morphological characteristics of the regolith in Baetica.

(6) The presence of the CBOE is ambiguous. The addition of the mechanism does not improve the fitting. We find that the SHOE parameters are robust for the tests conducted with and without CBOE. We thus conclude that shadow-hiding must be the dominant opposition effect mechanism, with an average HWHM of $4.6^{\circ}$.

(7) Even after photometric correction with two different approaches, Gades and Corduba remains collectively different from the surroundings on different ways. Gallicum Labes and Low Corduba are redder and composed of bright material exposed by nearby perturbation or impact. The Hapke parameters point to a porous regolith distribution of fine grains. The morphology in the region points to landslides with no further signs of craterization over it. To reinforce this assumption, we observe the bright structure $\mathbf{J}$ in Etruria. This structure is few pixels wide $(\sim 4 \mathrm{~km})$ and starts from a small crater and comes down to a deeper depression, as the filaments also found in Gallicum. However, we cannot certainly state that Gallicum is composed of a fresher material that has not suffered further space weathering or just a different composition.

Acknowledgements. The authors thanks CNPq, process no.402085/2012-4, for the support. FAPERJ and CAPES are also aknowledged for diverse grants and fellowships to D.L. and P.H.H.

OSIRIS was built by a consortium of the Max-Planck- Institut fi $i_{\zeta} \ltimes r$ Sonnensystemforschung, Gïckttingen, Germany, CISAS-University of Padova, Italy, the Laboratoire d'Astrophysique de Marseille, France, the Instituto de Astrofi; European Space Agency, Noordwijk, The Netherlands, the Instituto Nacional de Ti; $i_{i}$ cnica Aeroespacial, Madrid, Spain, the Universidad Politï ¿xchnica de Madrid, Spain, the Department of Physics and Astronomy of Uppsala University, Sweden, and the Institut fi氵 œr Datentechnik und Kommunikationsnetze der Technischen Universitï ¿at Braunschweig, Germany. The support of the national funding agencies of Germany (DLR), France (CNES), Italy (ASI), Spain (MEC), Sweden (SNSB), and the ESA Technical Directorate is gratefully acknowledged.

\section{REFERENCES}

Akimov, L. A. (1988), Light reflection by the moon. II, Kinematika i Fizika Nebesnykh Tel, 4, 10-16.

Akkermans, E., P. Wolf, R. Maynard, and G. Maret (1988), Theoretical study of the coherent backscattering of light by disordered media, Journal de Physique, 49, 77-98.

Barucci, M., F. M., J. J., M. S., and T. N. (in press), The Flybys of Asteroids 2867 Steins, 21 Lutetia, and 4179 Toutatis, Asteroids IV, editors: P.Michel, B. Bottke and F. DeMeo. 
Barucci, M. A., et al. (2005), Asteroid target selection for the new Rosetta mission baseline. 21 Lutetia and 2867 Steins, Astronomy and Astrophysics, 430, 313-317, doi:10.1051/ 0004-6361:20041505.

Barucci, M. A., et al. (2012), Overview of Lutetia's surface composition, planss, 66, $23-$ 30, doi:10.1016/j.pss.2011.11.009.

Beck, P., A. Pommerol, N. Thomas, B. Schmitt, F. Moynier, and J.-A. Barrat (2012), Photometry of meteorites, Icarus, 218, 364-377, doi:10.1016/j.icarus.2011.12.005.

Belskaya, I. N., V. G. Shevchenko, N. N. Kiselev, Y. N. Krugly, N. M. Shakhovskoy, Y. S. Efimov, N. M. Gaftonyuk, A. Cellino, and R. Gil-Hutton (2003), Opposition polarimetry and photometry of S- and E-type asteroids, Icarus, 166, 276-284, doi:10.1016/j.icarus. 2003.09.005.

Belskaya, I. N., S. Fornasier, Y. N. Krugly, V. G. Shevchenko, N. M. Gaftonyuk, M. A. Barucci, M. Fulchignoni, and R. Gil-Hutton (2010), Puzzling asteroid 21 Lutetia: our knowledge prior to the Rosetta fly-by, Astronomy and Astrophysics, 515, A29, doi:10. 1051/0004-6361/201013994.

Broyden, C. G. (1970), The Convergence of a Class of Double-rank Minimization Algorithms 1. General Considerations, IMA Journal of Applied Mathematics, 6(1), 76-90, doi:10.1093/imamat/6.1.76.

Capanna, C., L. Jorda, P. L. Lamy, and G. Gesquiere (2012), A new multiresolution method applied to the 3D reconstruction of small bodies, AGU Fall Meeting Abstracts, p. B1937.

Carry, B., et al. (2010), Physical properties of the ESA Rosetta target asteroid (21) Lutetia. II. Shape and flyby geometry, Astronomy and Astrophysics, 523, A94, doi: 10.1051/0004-6361/201015074.

Clark, B. E., P. Helfenstein, J. F. Bell, C. Peterson, J. Veverka, N. I. Izenberg, D. Domingue, D. Wellnitz, and L. McFadden (2002), NEAR Infrared Spectrometer Photometry of Asteroid 433 Eros, Icarus, 155, 189-204, doi:10.1006/icar.2001.6748.

Clark, B. E., et al. (1999), NEAR Photometry of Asteroid 253 Mathilde, Icarus, 140, 5365, doi:10.1006/icar.1999.6124.

Clark, B. E., et al. (2001), Space weathering on Eros: Constraints from albedo and spectral measurements of Psyche crater, Meteoritics and Planetary Science, 36, 1617-1637, doi: 10.1111/j.1945-5100.2001.tb01853.x.

Coradini, A., et al. (2011), The Surface Composition and Temperature of Asteroid 21 Lutetia As Observed by Rosetta/VIRTIS, Science, 334, 492-, doi:10.1126/science.1204062.

Déau, E., A. Flandes, L. J. Spilker, and J. Petazzoni (2013), Re-analysis of previous laboratory phase curves: 1 . Variations of the opposition effect morphology with the textural properties, and an application to planetary surfaces, Icarus, 226, 1465-1488, doi: 10.1016/j.icarus.2013.01.014.

Fairbairn, M. B. (2005), Planetary Photometry: The Lommel-Seeliger Law, jrasc, 99, 92.

Farnham, T. (2013), SHAPE MODEL OF ASTEROID 21 LUTETIA, RO-AOSINAC/OSIWAC-5-LUTETIA-SHAPE-V1.0, NASA Planetary Data System.

Fernando, J., F. Schmidt, X. Ceamanos, P. Pinet, S. Douté, and Y. Daydou (2013), Surface reflectance of Mars observed by CRISM/MRO: 2. Estimation of surface photometric properties in Gusev Crater and Meridiani Planum, Journal of Geophysical Research (Planets), 118, 534-559, doi:10.1029/2012JE004194.

Fernando, J., F. Schmidt, C. Pilorget, P. Pinet, X. Ceamanos, S. Douté, Y. Daydou, and F. Costard (2015a), Characterization and mapping of surface physical properties of Mars from CRISM multi-angular data: Application to Gusev Crater and Meridiani Planum, Icarus, 253, 271-295, doi:10.1016/j.icarus.2015.03.012. 
Fernando, J., F. Schmidt, C. Pilorget, P. Pinet, X. Ceamanos, S. Douté, Y. Daydou, and F. Costard (2015b), Characterization and mapping of surface physical properties of mars from \{CRISM \} multi-angular data: Application to gusev crater and meridiani planum, Icarus, 253(0), 271 - 295, doi:http://dx.doi.org/10.1016/j.icarus.2015.03.012.

Fornasier, S., et al. (2015), Spectrophotometric properties of the nucleus of comet 67P/Churyumov-Gerasimenko from the OSIRIS instrument onboard the ROSETTA spacecraft, ArXiv e-prints.

Gaffey, M. J. (2010), Space weathering and the interpretation of asteroid reflectance spectra, Icarus, 209, 564-574, doi:10.1016/j.icarus.2010.05.006.

Gehrels, T. (1956), Photometry of asteroids., Astrophysical Journal, 61, 178, doi:10.1086/ 107312.

Goguen, J. D., T. C. Stone, H. H. Kieffer, and B. J. Buratti (2010), A new look at photometry of the Moon, Icarus, 208, 548-557, doi:10.1016/j.icarus.2010.03.025.

Gulkis, S., et al. (2012), Continuum and spectroscopic observations of asteroid (21) Lutetia at millimeter and submillimeter wavelengths with the MIRO instrument on the Rosetta spacecraft, planss, 66, 31-42, doi:10.1016/j.pss.2011.12.004.

Hapke, B. (1981), Bidirectional reflectance spectroscopy. 1. Theory, Journal of Geophysical Research, 86, 4571-4586.

Hapke, B. (1984), Bidirectional reflectance spectroscopy. III - Correction for macroscopic roughness, Icarus, 59, 41-59, doi:10.1016/0019-1035(84)90054-X.

Hapke, B. (1993), Theory of reflectance and emittance spectroscopy.

Hapke, B. (2002), Bidirectional Reflectance Spectroscopy. 5. The Coherent Backscatter Opposition Effect and Anisotropic Scattering, Icarus, 157, 523-534, doi:10.1006/icar. 2002.6853.

Hapke, B. (2008), Bidirectional reflectance spectroscopy. 6. Effects of porosity, Icarus, 195, 918-926, doi:10.1016/j.icarus.2008.01.003.

Hapke, B. (2012), Theory of Reflectance and Emittance Spectroscopy, second ed., Cambridge University Press, cambridge Books Online.

Helfenstein, P., and M. K. Shepard (2011), Testing the Hapke photometric model: Improved inversion and the porosity correction, Icarus, 215, 83-100, doi:10.1016/j.icarus. 2011.07.002.

Helfenstein, P., and J. Veverka (1989), Physical characterization of asteroid surfaces from photometric analysis, in Asteroids II, edited by R. P. Binzel, T. Gehrels, and M. S. Matthews, pp. 557-593.

Helfenstein, P., et al. (1994), Galileo photometry of Asteroid 951 Gaspra, Icarus, 107, 37, doi:10.1006/icar.1994.1005.

Helfenstein, P., et al. (1996), Galileo Photometry of Asteroid 243 Ida, Icarus, 120, 48-65, doi:10.1006/icar.1996.0036.

Hillier, J. K., J. M. Bauer, and B. J. Buratti (2011), Photometric modeling of Asteroid 5535 Annefrank from Stardust observations, Icarus, 211, 546-552, doi:10.1016/j.icarus.2010. 10.009 .

Jorda, L., R. Gaskell, P. Lamy, M. Kaasalainen, O. Groussin, G. Faury, P. Gutierrez, W. Sabolo, and S. Hviid (2011), Shape and Physical Properties of Asteroid (21) Lutetia from OSIRIS Images, in EPSC-DPS Joint Meeting 2011, p. 776.

Keller, H. U., et al. (2007), Observations of Comet 9P/Tempel 1 around the Deep Impact event by the OSIRIS cameras onboard Rosetta, Icarus, 187, 87-103, doi: 10.1016/j.icarus.2006.09.023. 
Kitazato, K., et al. (2008), Near-infrared spectrophotometry of Asteroid 25143 Itokawa from NIRS on the Hayabusa spacecraft, Icarus, 194, 137-145, doi:10.1016/j.icarus. 2007.08.029.

Levenberg, K. (1944), A Method for the Solution of Certain Non-Linear Problems in Least Squares, Quarterly of Applied Mathematics, 2, 164-168.

Leyrat, C., et al. (2010), Search for Steins surface inhomogeneities from OSIRIS Rosetta images, planss, 58, 1097-1106, doi:10.1016/j.pss.2010.04.003.

Li, J., M. F. A'Hearn, and L. A. McFadden (2004), Photometric analysis of Eros from NEAR data, Icarus, 172, 415-431, doi:10.1016/j.icarus.2004.07.024.

Li, J.-Y., L. A. McFadden, J. W. Parker, E. F. Young, S. A. Stern, P. C. Thomas, C. T. Russell, and M. V. Sykes (2006), Photometric analysis of 1 Ceres and surface mapping from HST observations, Icarus, 182, 143-160, doi:10.1016/j.icarus.2005.12.012.

Li, J.-Y., M. F. A'Hearn, L. A. McFadden, and M. J. S. Belton (2007a), Photometric analysis and disk-resolved thermal modeling of Comet 19P/Borrelly from Deep Space 1 data, Icarus, 188, 195-211, doi:10.1016/j.icarus.2006.11.015.

Li, J.-Y., M. F. A'Hearn, T. L. Farnham, and L. A. McFadden (2009), Photometric analysis of the nucleus of Comet 81P/Wild 2 from Stardust images, Icarus, 204, 209-226, doi: 10.1016/j.icarus.2009.06.002.

Li, J.-Y., et al. (2007b), Deep Impact photometry of Comet 9P/Tempel 1, Icarus, 187, 41-55, doi:10.1016/j.icarus.2006.09.018.

Li, J.-Y., et al. (2013a), Photometric properties of the nucleus of Comet 103P/Hartley 2, Icarus, 222, 559-570, doi:10.1016/j.icarus.2012.11.001.

Li, J.-Y., et al. (2013b), Global photometric properties of Asteroid (4) Vesta observed with Dawn Framing Camera, Icarus, 226, 1252-1274, doi:10.1016/j.icarus.2013.08.011.

Lumme, K., J. I. Peltoniemi, and W. M. Irvine (1990), Diffuse reflection from a stochastically bounded, semi-infinite medium, Transport Theory and Statistical Physics, 19, 317-332, doi:10.1080/00411459008203894.

Magrin, S., et al. (2012), (21) Lutetia spectrophotometry from Rosetta-OSIRIS images and comparison to ground-based observations, planss, 66, 43-53, doi:10.1016/j.pss.2011.10. 001.

Marchi, S., et al. (2012), The cratering history of asteroid (21) Lutetia, planss, 66, 87-95, doi:10.1016/j.pss.2011.10.010.

Masoumzadeh, N., H. Boehnhardt, J.-Y. Li, and J.-B. Vincent (2015), Photometric analysis of Asteroid (21) Lutetia from Rosetta-OSIRIS images, Icarus, 257, 239-250, doi:10. 1016/j.icarus.2015.05.013.

McEwen, A. S. (1991), Photometric functions for photoclinometry and other applications, Icarus, 92, 298-311, doi:10.1016/0019-1035(91)90053-V.

McEwen, A. S. (1996), A Precise Lunar Photometric Function, in Lunar and Planetary Science Conference, Lunar and Planetary Science Conference, vol. 27, p. 841.

McGuire, A. F., and B. W. Hapke (1995), An experimental study of light scattering by large, irregular particles, Icarus, 113, 134-155, doi:10.1006/icar.1995.1012.

Minnaert, M. (1941), The reciprocity principle in lunar photometry, Astrophysical Journal, 93, 403-410, doi:10.1086/144279.

Mishchenko, M. I., J. M. Dlugach, L. Liu, V. K. Rosenbush, N. N. Kiselev, and Y. G. Shkuratov (2009), Direct Solutions of the Maxwell Equations Explain Opposition Phenomena Observed for High-Albedo Solar System Objects, Astrophysical Journal, 705, L118-L122, doi:10.1088/0004-637X/705/2/L118. 
More, J. (1978), The levenberg-marquardt algorithm: Implementation and theory, in $\mathrm{Nu}$ merical Analysis, Lecture Notes in Mathematics, vol. 630, edited by G. Watson, pp. 105-116, Springer Berlin Heidelberg, doi:10.1007/BFb0067700.

Muinonen, K. (1994), Coherent Backscattering by Solar System Dust Particles, in Asteroids, Comets, Meteors 1993, IAU Symposium, vol. 160, edited by A. Milani, M. di Martino, and A. Cellino, p. 271.

Muinonen, K., M. I. Mishchenko, J. M. Dlugach, E. Zubko, A. Penttilä, and G. Videen (2012), Coherent Backscattering Verified Numerically for a Finite Volume of Spherical Particles, Astrophysical Journal, 760, 118, doi:10.1088/0004-637X/760/2/118.

Oren, M., and S. K. Nayar (1993), Diffuse Reflectance from Rough Surfaces, in IEEE Conference on Computer Vision and Pattern Recognition (CVPR), pp. 763-764.

O'Rourke, L., et al. (2012), Thermal and shape properties of asteroid (21) Lutetia from Herschel observations around the Rosetta flyby, planss, 66, 192-199, doi:10.1016/j.pss. 2012.01.004.

Pätzold, M., et al. (2011), Asteroid 21 Lutetia: Low Mass, High Density, Science, 334, 491-, doi:10.1126/science.1209389.

Rosenbush, V. K., V. G. Shevchenko, N. N. Kiselev, A. V. Sergeev, N. M. Shakhovskoy, F. P. Velichko, S. V. Kolesnikov, and N. V. Karpov (2009), Polarization and brightness opposition effects for the E-type Asteroid 44 Nysa, Icarus, 201, 655-665, doi:10.1016/ j.icarus.2009.01.007.

Sato, H., M. S. Robinson, B. Hapke, B. W. Denevi, and A. K. Boyd (2014), Resolved Hapke parameter maps of the Moon, Journal of Geophysical Research (Planets), 119, 1775-1805, doi:10.1002/2013JE004580.

Schmidt, F., and J. Fernando (2015), Realistic uncertainties on Hapke model parameters from photometric measurement, Icarus, 260, 73-93, doi:10.1016/j.icarus.2015.07.002.

Schröder, S. E., S. Mottola, H. U. Keller, C. A. Raymond, and C. T. Russell (2013), Resolved photometry of Vesta reveals physical properties of crater regolith, planss, 85, 198-213, doi:10.1016/j.pss.2013.06.009.

Schröder, S. E., Y. Grynko, A. Pommerol, H. U. Keller, N. Thomas, and T. L. Roush (2014), Laboratory observations and simulations of phase reddening, Icarus, 239, 201216, doi:10.1016/j.icarus.2014.06.010.

Schulz, R., H. Sierks, M. Küppers, and A. Accomazzo (2012), Rosetta fly-by at asteroid (21) Lutetia: An overview, planss, 66, 2-8, doi:10.1016/j.pss.2011.11.013.

Shepard, M. K., and P. Helfenstein (2007), A test of the Hapke photometric model, Journal of Geophysical Research (Planets), 112, E03001, doi:10.1029/2005JE002625.

Shepard, M. K., and P. Helfenstein (2011), A laboratory study of the bidirectional reflectance from particulate samples, Icarus, 215, 526-533, doi:10.1016/j.icarus.2011.07. 033.

Shkuratov, Y., V. Kaydash, V. Korokhin, Y. Velikodsky, N. Opanasenko, and G. Videen (2011), Optical measurements of the Moon as a tool to study its surface, planss, 59, 1326-1371, doi:10.1016/j.pss.2011.06.011.

Shkuratov, Y., V. Kaydash, V. Korokhin, Y. Velikodsky, D. Petrov, E. Zubko, D. Stankevich, and G. Videen (2012), A critical assessment of the Hapke photometric model, Journal of Quantitative Spectroscopy and Radiative Transfer, 113, 2431-2456, doi: 10.1016/j.jqsrt.2012.04.010.

Shkuratov, Y., et al. (2002), The Opposition Effect and Negative Polarization of Structural Analogs for Planetary Regoliths, Icarus, 159, 396-416, doi:10.1006/icar.2002.6923. 
Shkuratov, Y. G., et al. (1994), A critical review of theoretical models of negatively polarized light scattered by atmosphereless solar system bodies, Earth Moon and Planets, 65, 201-246, doi:10.1007/BF00579535.

Sierks, H., et al. (2011), Images of Asteroid 21 Lutetia: A Remnant Planetesimal from the Early Solar System, Science, 334, 487-, doi:10.1126/science.1207325.

Simonelli, D. P., M. Wisz, A. Switala, D. Adinolfi, J. Veverka, P. C. Thomas, and P. Helfenstein (1998), Photometric Properties of PHOBOS Surface Materials From Viking Images, Icarus, 131, 52-77, doi:10.1006/icar.1997.5800.

Spjuth, S., L. Jorda, P. L. Lamy, H. U. Keller, and J.-Y. Li (2012), Disk-resolved photometry of Asteroid (2867) Steins, Icarus, 221, 1101-1118, doi:10.1016/j.icarus.2012.06. 021.

Stankevich, D. G., Y. G. Shkuratov, and K. Muinonen (1999), Shadow-hiding effect in inhomogeneous layered particulate media., Journal of Quantitative Spectroscopy and Radiative Transfer, 63, 445-458, doi:10.1016/S0022-4073(99)00030-8.

Thomas, N., et al. (2012), The geomorphology of (21) Lutetia: Results from the OSIRIS imaging system onboard ESA's Rosetta spacecraft, planss, 66, 96-124, doi:10.1016/j. pss.2011.10.003.

Thomas, P. C., D. Adinolfi, P. Helfenstein, D. Simonelli, and J. Veverka (1996), The Surface of Deimos: Contribution of Materials and Processes to Its Unique Appearance, Icarus, 123, 536-556, doi:10.1006/icar.1996.0177.

Tubiana, C., et al. (2015), 67P/Churyumov-Gerasimenko: Activity between March and June 2014 as observed from Rosetta/OSIRIS, Astronomy and Astrophysics, 573, A62, doi:10.1051/0004-6361/201424735.

Van Der Walt, S., S. C. Colbert, and G. Varoquaux (2011), The numpy array: A structure for efficient numerical computation, Computing in Science \& Engineering, 13(2), 2230, doi:http://dx.doi.org/10.1109/MCSE.2011.37.

Zhu, C., R. H. Byrd, P. Lu, and J. Nocedal (1997), Algorithm 778: L-bfgs-b: Fortran subroutines for large-scale bound-constrained optimization, ACM Trans. Math. Softw., 23(4), 550-560, doi:10.1145/279232.279236. 

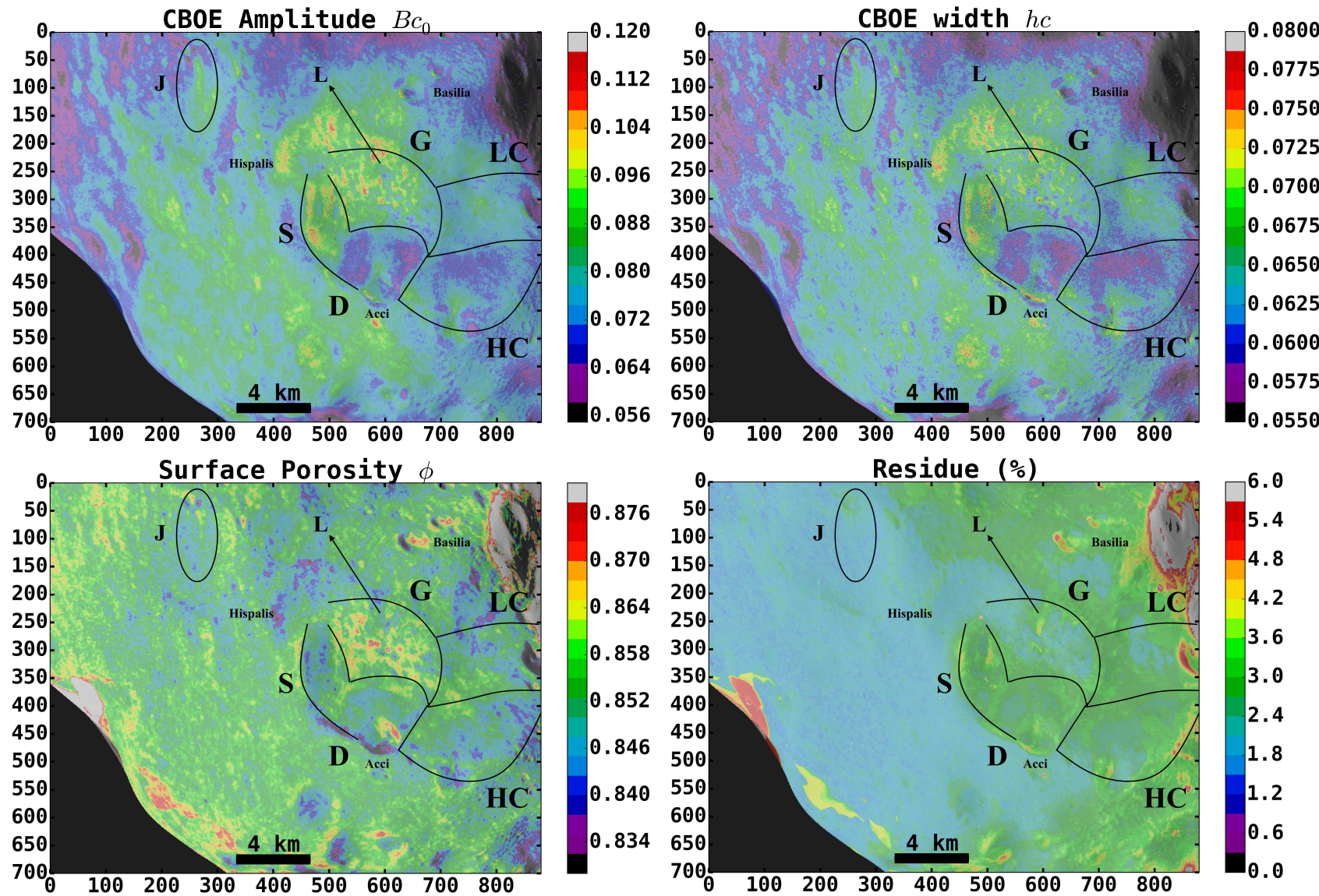

Residue (\%)
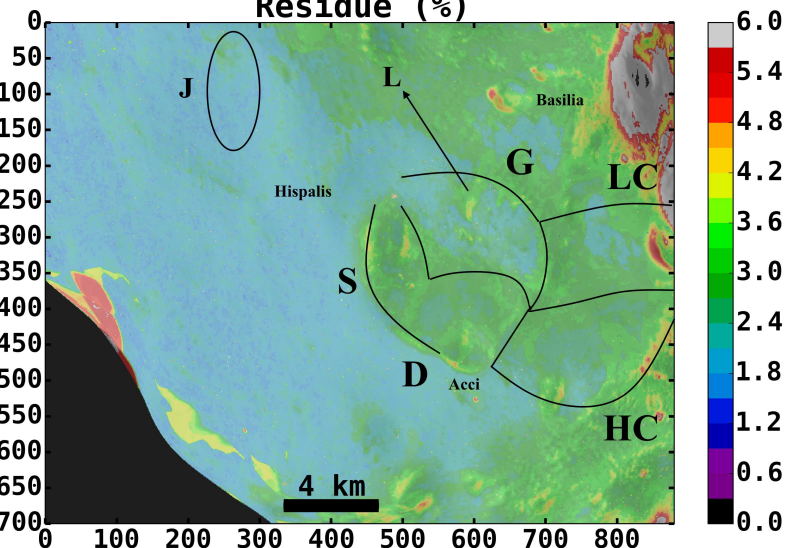

Cont. Figure 4.2. 


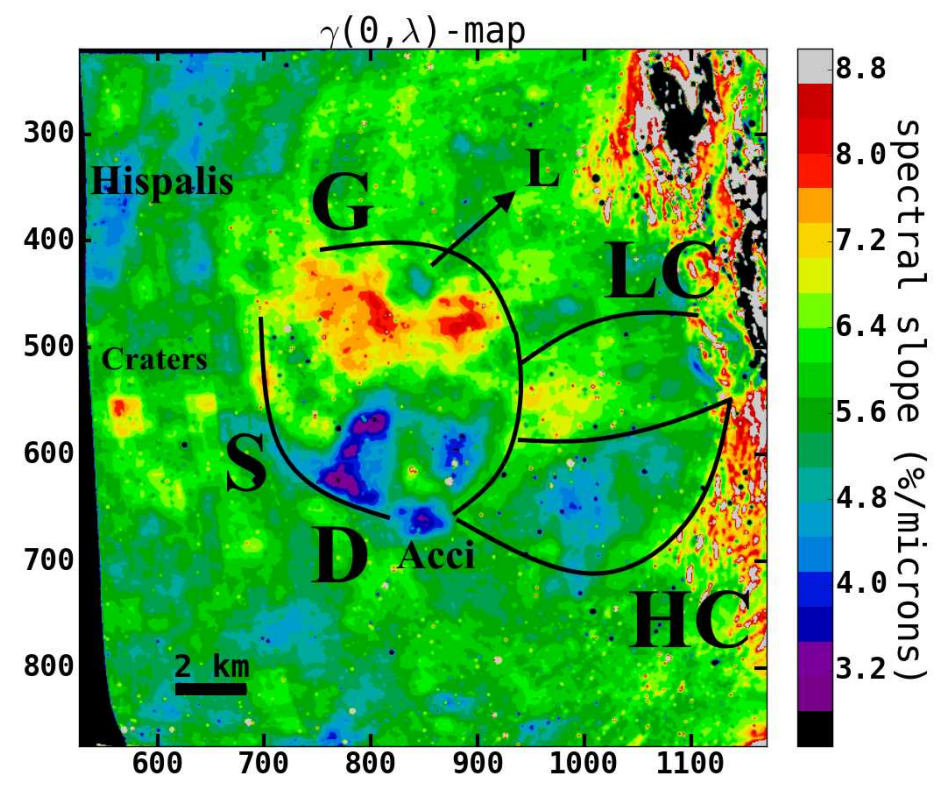

(A)

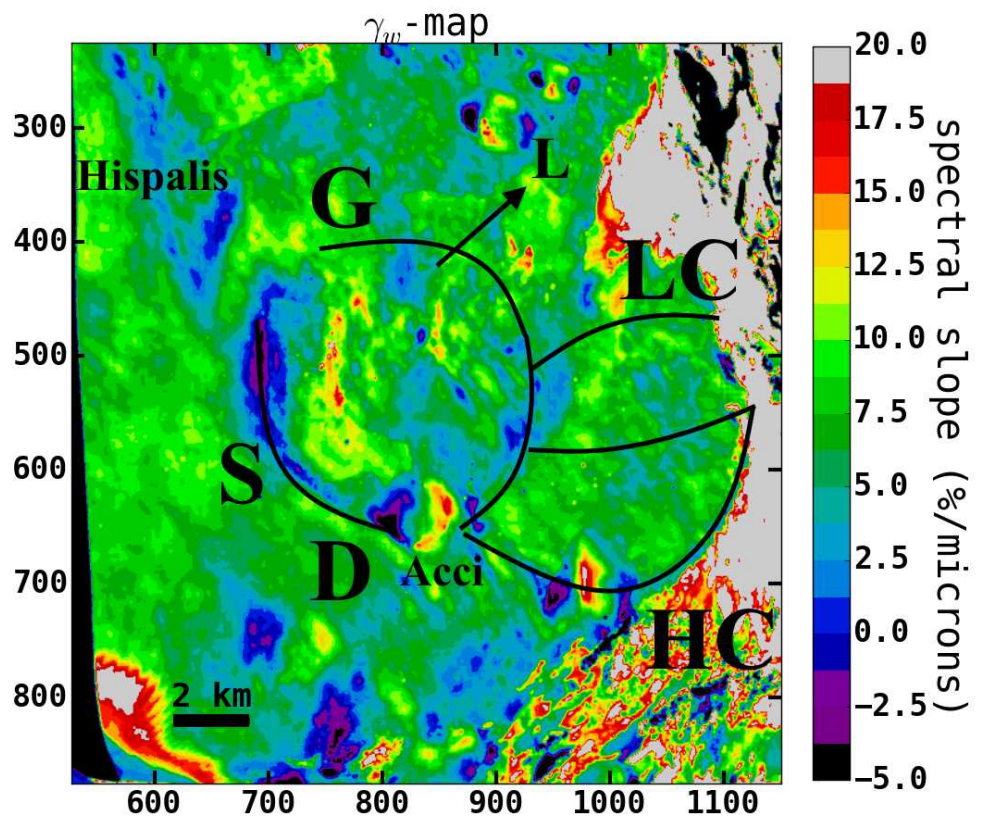

(B)

FIGURE 4.3. Spectrophotometric slope $\gamma$ of (a) the Normal Albedo and (b) the single-scattering albedo. Projected into NAC_2010-0710T15.40.39.231Z_ID30_1251276000_F82 image. (G) Gallicum. (D) Danuvius. (S) Sarnus. (HC) High Corduba. (LC) Low Corduba. 

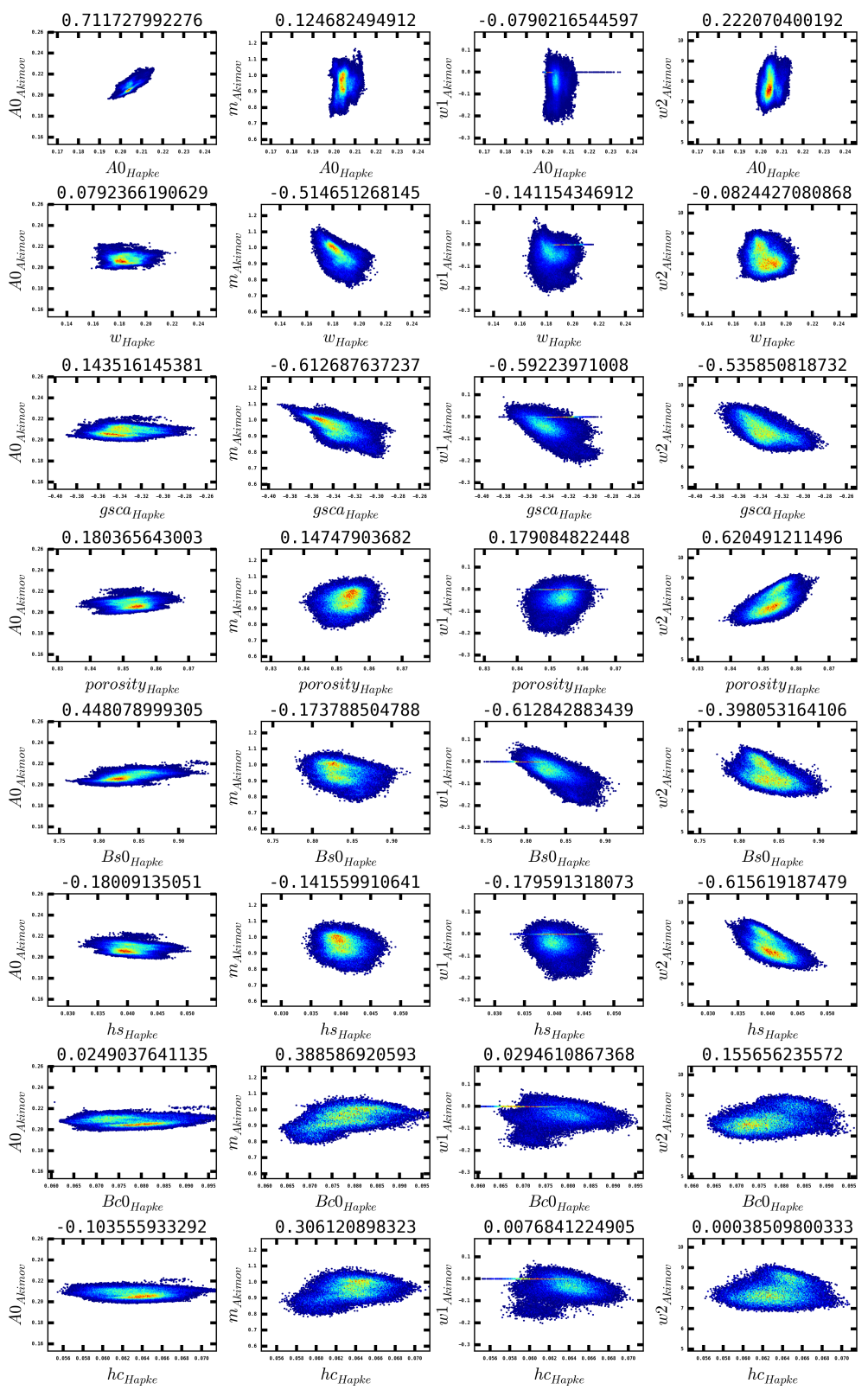

FiguRE 5.1. Correlation of pixels of the projected map in the NAC_2010-07-10T15.40.39.231Z_ID30_1251276000_F82 image for Akimov phase parameters and Hapke parameters in the NAC F82+22 filter. The parameters are calculated for each facet then translated to pixels according to the methodology described on subsections 3.1 and 4.2. The title on each plot references to the respective Sperman's rank correlation coefficient. 